\title{
A General Route Toward the Synthesis of the Cladiellin Skeleton Utilizing a Sml 2 -Mediated Cyclization
}

\author{
Gary A. Molander ${ }^{*}$ Barbara Czakó, and David J. St. Jean Jr. \\ Roy and Diana Vagelos Laboratories, Department of Chemistry, University of Pennsylvania, \\ Philadelphia, Pennsylvania 19104-6323
}

\begin{abstract}
An efficient synthesis of the cladiellin skeleton is reported utilizing methods that were previously developed in this laboratory. The approach is based on a $\mathrm{SmI}_{2}$-mediated cyclization reaction for the construction of the oxacyclononane unit. A [4+3] annulation strategy was used to create the octahydroisobenzofuran moiety. This route provides the cladiellin skeleton in only fourteen steps without the use of protecting groups. The present approach also enabled the synthesis of the 3,7diastereomer of the natural product polyanthellin A.
\end{abstract}

\section{Keywords}

cladiellin skeleton; $\mathrm{SmI}_{2}$-mediated cyclization

\section{Introduction}

Cladiellin diterpenes belong to a family of marine metabolites isolated from gorgonians and soft corals. ${ }^{1}$ The first member of this family, eunicellin, was isolated by Djerassi and coworkers in 1968, and since then more than sixty cladiellin natural products have been reported. ${ }^{1,2}$ Cladiellins possess unique structural features: a rare oxabicyclic ring system that is composed of an octahydroisobenzofuran moiety and an oxacyclononane unit (Figure 1). ${ }^{1}$ Furthermore, these molecules contain at least six stereogenic centers. In addition to their intriguing structure, several members of this family exhibit interesting biological activity such as cytotoxic activity, anti-inflammatory effects and antimalarial properties. ${ }^{1}$ The challenging structure of these compounds, coupled with their diverse biological properties, has made them attractive synthetic targets for several research groups. $3-13$

Construction of medium-sized ring compounds is often problematic, and there are only a limited number of methods available for creating these systems. It was demonstrated previously that medium-sized rings can be efficiently synthesized by $\mathrm{SmI}_{2}$-mediated cyclization. ${ }^{14-16}$ The focus of the present research was to devise a concise route toward the synthesis of the cladiellin skeleton wherein a $\mathrm{SmI}_{2}$-mediated cyclization was utilized to create the oxacyclononane subunit. For constructing the octahydroisobenzofuran moiety, a [4+3] annulation reaction was employed. ${ }^{13,17}$

\section{Results and Discussion}

The initial synthetic approach is depicted in Scheme 1. The strategy was based on a $\mathrm{SmI}_{2}-$ mediated acyl substitution reaction, a method that was developed and widely studied in this 


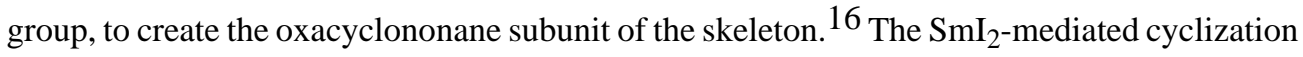
precursor $\mathbf{2}$ was envisioned to derive from hydroxy lactone $\mathbf{3}$ that in turn could be derived from tricyclic ketone $\mathbf{4}$ via a Baeyer-Villiger oxidation. The tertiary acetoxy functionality at the C-11 position is prevalent in several cladiellin diterpene natural products, and this functional group was to be introduced at this stage of the synthesis. Compound 5 could be obtained from [4+3] annulation product 6 .

The initial steps of the sequence are depicted in Scheme 2. The [4+3] annulation product 6 was synthesized following a previously developed procedure. ${ }^{13}$ According to this route, compound 6 could be obtained in only three steps from commercially available $(R)-(-)-\alpha$-phellandrene. First, $(R)-(-)-\alpha$-phellandrene was reacted in a $[2+2]$ ketene-olefin cycloaddition. 18 This reaction was followed by a photorearrangement to provide bis-acetal $8 .{ }^{19-21}$ Tricycle 6 could be formed by a $\mathrm{TiCl}_{4}$-mediated [4+3] annulation reaction between bis-acetal $\mathbf{8}$ and 1methoxy-1,3-bis(triethylsilanyloxy)-1,3-butadiene. ${ }^{17}$ Subsequent methylation of the dianion derived from the [4+3] annulation product 6, Krapcho decarboxylation, and epimerization led to the desired methyl ketone $9 .{ }^{13,22}$ The next task was to create the quaternary carbon stereocenter alpha to the carbonyl. Direct allylation of the anion derived from methyl ketone 9 proved to be problematic due to decomposition of the substrate under various conditions. Eventually, this transformation could be realized in a two step sequence. Treatment of $\alpha$-methyl ketone 9 with potassium hydride and tert-butyldimethylsilyl chloride provided the thermodynamic silyl enol ether with high regioselectivity. Introduction of the allyl group was achieved via a silver trifluoroacetate-mediated allylation using allyl iodide. ${ }^{23}$ Subsequently, the primary hydroxyl functionality was formed through a chemoselective hydroboration of the terminal alkene with dicyclohexylborane in the presence of the trisubstituted double bond and the ketone providing compound $\boldsymbol{5}^{24,25}$

Previous studies had indicated that some compounds containing the 8-oxabicylo[3.2.1]octa-3one subunit, as in structure 5, do not undergo Baeyer-Villiger oxidation readily. ${ }^{26}$ However, the viability of this transformation was tested again using compound 5. Several oxidizing agents were tried ( $m$-CPBA, magnesium monoperoxyphthalate, trifluoroperacetic acid, peracetic acid and bis(trimethylsilyl) peroxide) under a variety of conditions (changing the temperature, applying buffers and radical scavengers), but in all instances only epoxidation occurred and no traces of the Baeyer-Villiger oxidation product could be isolated (Scheme 3). ${ }^{27}$

Simultaneously, studies on model systems were also conducted (Scheme 4). Interestingly, applying trifluoroperacetic acid (TFPAA) generated from trifluoroacetic anhydride (TFAA) and $90 \% \mathrm{H}_{2} \mathrm{O}_{2}$ on model ketone 9 led to the desired lactone $\mathbf{1 3}$, while no lactone formation occurred on ketone 14. ${ }^{28}$

These results may imply that the rate determining step of this Baeyer-Villiger oxidation is the nucleophilic attack of the trifluoroperoxy acid to the ketone. ${ }^{29,30}$ The outcome of the model studies could thus be explained by examining the three-dimensional structures of compounds 9 and 14 (Figure 2). In both cases, nucleophilic addition of trifluoroperacetic acid to the ketone is expected to occur from the less hindered convex face of the ring system. The 3D structures of compound $\mathbf{9}$ and $\mathbf{1 4}$ reveal that in the case of compound $\mathbf{9}$, where the methyl substituent is on the concave face, the attack of the peracid can take place without difficulty. However, when the methyl group is situated on the convex side of the ring system, as in compound 14, the ketone is sterically encumbered, thus the attack of the peracid is hindered. (Figure 2).

The above findings prompted a revision of the synthetic strategy. The modified approach, shown in Scheme 5, diverged from the original synthesis after the [4+3] annulation. In this route, the allyl side chain was introduced onto the annulation product 6 directly. Subsequent functionalization would provide the required Baeyer-Villiger oxidation precursor 19. As in the 
previous approach, the construction of the oxacyclononane ring system was envisioned via a $\mathrm{SmI}_{2}$-mediated acyl substitution.

The synthetic sequence investigating this approach is depicted in Scheme 6. The route started with the allylation of the [4+3] annulation product 6 using $n$-BuLi and allyl iodide, providing the product as the only diastereomer. Subsequent Krapcho decarboxylation and epimerization led to a 3:1 mixture of compound $\mathbf{2 1}$ and its epimer. After separation, the minor diastereomer could be recycled and epimerized to the desired product. 22 Selective hydroboration/oxidation could be achieved as before using dicyclohexylborane, 24,25 and the resulting alcohol was easily protected as a tert-butyldimethylsilyl ether. ${ }^{31}$ Introduction of the tertiary acetate group was realized in a three step sequence providing compound 19. First, halohydrin formation was achieved using $N$-bromosuccinimide and water. ${ }^{32}$ Removal of the bromide was accomplished in a radical reaction utilizing tributyltin hydride. ${ }^{33,34}$ Subsequent acylation proved to be problematic due to the propensity of the tertiary alcohol to eliminate. After extensive optimization it was found that this transformation could be effected using acetic anhydride, triethylamine, and substoichiometric quantities of DMAP, providing the Baeyer-Villiger oxidation precursor. ${ }^{35}$ Gratifyingly, Baeyer-Villiger oxidation of compound 19 readily occurred using $90 \%$ trifluoroperacetic acid. ${ }^{27}$ Further optimization of the oxidation was attempted using $m$-chloroperbenzoic acid, magnesium monoperoxyphthalate, and peracetic acid under various conditions, but trifluoroperacetic acid proved to be the most efficient oxidant. Subsequent formation of the cyclization precursor $\mathbf{1 7}$ was realized in two steps, first by removing the TBDPS protecting group using TBAF and forming the corresponding iodide by triphenylphosphine and iodine. 31,36

With compound 17 in hand, the $\mathrm{SmI}_{2}$-mediated acyl substitution was attempted in the presence of HMPA or $\mathrm{NiI}_{2}$ (Scheme 7). ${ }^{16,37}$ However, these transformations led only to reduced material and no cyclization product could be observed.

The outcome of this reaction can be rationalized by unfavorable conformational effects. In the minimum energy conformation, the organosamarium side chain is in a quasi-equatorial orientation. ${ }^{38}$ In order for the transformation to occur, this side chain must rotate into a quasiaxial orientation, and the attack on the lactone needs to occur from the concave face of the ring system (Figure 3). Based on MMFF calculations, the energy difference between the minimum energy conformation and the conformation where the sidechain is in a quasi-axial orientation is $8.05 \mathrm{kcal} / \mathrm{mol}$.

As the $\mathrm{SmI}_{2}$-mediated acyl substitution did not lead to the desired product, a third approach was devised, which also included a $\mathrm{SmI}_{2}$-mediated cyclization. However, the cyclization was planned to be performed on the more reactive and sterically more feasible iodo ketone $\mathbf{2 6}$ (Scheme 8). The cladiellin skeleton would then be obtained via elimination and oxidative cleavage of the resulting double bond.

To arrive at the cyclization precursor 26, the sequence began with compound 27, a known intermediate in the previous approach (Scheme 9). First, selective hydroboration of monosubstituted alkene was carried out with dicyclohexylborane. ${ }^{24,25}$ To avoid protecting group manipulations, the primary alcohol was transformed into the corresponding alkyl chloride using hexachloroacetone and triphenylphosphine. ${ }^{39}$ Introduction of the tertiary acetate could be achieved as before, in a three step sequence. ${ }^{32-35}$ To form the $\mathrm{SmI}_{2}$-mediated cyclization precursor, the alkyl chloride was transformed to the corresponding alkyl iodide 26 via a Finkelstein reaction. ${ }^{40} \mathrm{Gratifyingly}$, the key $\mathrm{SmI}_{2}$-mediated Barbier-reaction provided compound 25 in excellent yield. ${ }^{15}$ Regioselective syn elimination of the tertiary alcohol could be effected using the Burgess reagent to give compound $\mathbf{2 4}$ as the only product in high yield. 41 Ozonolysis of the tetrasubstituted double bond provided the desired product 23 , a compound 
that incorporates all of the carbon atoms of the cladiellin skeleton. ${ }^{42}$ With this transformation, the goal of constructing the cladiellin core was achieved.

Having synthesized the skeleton of the cladiellin natural products, the challenge to differentiate between the two carbonyl functionalities of compound $\mathbf{2 3}$ remained. Studies conducted on compound 23 indicated that a Wittig reaction, carried out under carefully controlled conditions, occurs with very high selectivity at the C-3 carbonyl providing compound $\mathbf{2 9}$ (Scheme 10).

43 The structure of the product was unambiguously demonstrated by 2D-NMR experiments. The HMBC spectrum of molecule 29 shows a correlation between H-2 and C-3, H-2 and C-18, and $\mathrm{H}-18$ and C-2 that clearly indicates the position of the double bond.

Several cladiellin natural products possess a tertiary alcohol at the C-7 position. Having compound 29 in hand, the formation of this functionality via methyl addition to the C-7 ketone was examined, devoting special attention to the diastereoselectivity of the transformation. Based on the crystal structure of diketone $\mathbf{2 3}$, as well as the minimum energy conformation of keto-alkene 29 (Figure 4), ${ }^{13,44}$ addition was expected to occur from the more open convex face or "outside" of the ring system, providing the stereochemistry desired for several target structures.

Initial studies showed that methyl addition to the carbonyl could not be realized by methyllithium or methylmagnesium bromide. In these cases, only starting material was recovered, perhaps owing to enolization of the carbonyl. However, by using $\mathrm{MeLi} / \mathrm{Yb}(\mathrm{OTf})_{3}$ efficient carbonyl addition was effected. ${ }^{45}$ Unfortunately, the stereochemistry of the resulting product could not be unambiguously proven by analysis of NOESY data of compound 30 . To elucidate the stereochemistry, the tertiary alcohol was transformed into compound $\mathbf{3 1}$ via oxymercuration with mercury trifluoroacetate and subsequent reduction. 46

The structure of the final product was established based on 2D-NMR experiments. To our surprise, the unanticipated diastereomer was formed in high yield. Proton and carbon assignments were made based on COSY, HMQC, and HMBC experiments, and the stereochemistry was confirmed based on NOESY experiments. The characteristic correlation between $\mathrm{H}-2$ and $\mathrm{CH}_{3}-18$, and $\mathrm{H}-8 \alpha$ and $\mathrm{CH}_{3}-19$, and the absence of correlations between $\mathrm{H}-1$ and $\mathrm{CH}_{3}-18$ and $\mathrm{H}-10$ and $\mathrm{CH}_{3}-19$ in the NOESY spectrum unambiguously established the stereochemistry of the product (Figure 5). These results indicated that methyl addition occurred from a different conformation than that predicted on the basis of the calculated and crystal structures, suggesting that in the reactive conformation chelation between the oxygen of the tetrahydrofuran ring and the ketone might play a key role. Unfortunately, addition of a polar, aprotic solvent such as HMPA to the $\mathrm{MeLi} / \mathrm{Yb}(\mathrm{OTf})_{3}$ reagent, which would prevent chelate formation, again resulted in the recovery of starting material.

Compound $\mathbf{3 1}$ is the 3,7-diastereomer of a cladiellin natural product, polyanthellin A (Figure 6) that was isolated from the gorgonian Briareum polyanthes in 2002. The synthesis of this compound was achieved in only 17 overall steps without the use of protecting groups from commercially available $(R)-(-)-\alpha$-phellandrene.

\section{Conclusion}

An efficient approach to the synthesis of the cladiellin skeleton was devised, featuring two methods that were previously developed in our research group. The medium-sized oxacyclononane subunit of the skeleton was created by a $\mathrm{SmI}_{2}$-mediated cyclization. 15 The octahydroisobenzofuran moiety was synthesized via a [4+3]-annulation method. ${ }^{13,17}$ Following this strategy, the cladiellin skeleton could be synthesized in only fourteen steps without the use of protecting groups. This route also enabled the synthesis of the 3,7diastereomer of polyanthellin A. 


\title{
Experimental Section
}

\section{Preparation of $(1 R, 2 R, 3 R, 7 S, 8 R, 11 R)$-11-allyl-10-hydroxy-3-isopropyl-6-methyl-12-oxa- tricyclo[6.3.1.0 2,7] dodeca-5,9-diene-9-carboxylic acid methyl ester (33)}

\begin{abstract}
A flask containing $\mathrm{LiCl}(4.9 \mathrm{~g}, 116.4 \mathrm{mmol}, 20$ equiv) was flame-dried three times. After the salt was cooled to rt under an argon, a solution of compound $20(1.7 \mathrm{~g}, 8.2 \mathrm{mmol})$ in THF (58 $\mathrm{mL}$ ) was added. Subsequently, the solution was cooled to $-78^{\circ} \mathrm{C}$ and $n$ - BuLi (2.5 M in hexane, $5 \mathrm{~mL}, 12.5 \mathrm{mmol}, 2.15$ equiv) was added dropwise. The reaction was stirred at this temperature for $35 \mathrm{~min}$. This process was followed by the dropwise addition of allyl iodide ( $793 \mu \mathrm{L}, 8.7$ mmol, 1.5 equiv). The reaction was allowed to warm to $-50{ }^{\circ} \mathrm{C}$ and stirred for $40 \mathrm{~min}$. The reaction was quenched with a saturated ammonium chloride solution $(20 \mathrm{~mL})$ at $-50{ }^{\circ} \mathrm{C}$. After warming to rt, the mixture was diluted with $\mathrm{Et}_{2} \mathrm{O}$ and the phases were separated. The aqueous phase was extracted with EtOAc $(3 \times 10 \mathrm{~mL})$. The combined organic phases were washed with a concentrated $\mathrm{NaCl}$ solution, dried over $\mathrm{MgSO}_{4}$ and the solvent was evaporated. The residue was purified via column chromatography applying EtOAc-hexane (3\%) as eluent to provide compound 33 (1.75 g, 90\%). ${ }^{1} \mathrm{H}-\mathrm{NMR}\left(500 \mathrm{MHz}, \mathrm{CDCl}_{3}\right): \delta 10.8(\mathrm{~s}, 1 \mathrm{H}), 5.86(\mathrm{~m}, 1 \mathrm{H}), 5.52$ (s, 1H), $5.12(\mathrm{~d}, J=16.2 \mathrm{~Hz}, 1 \mathrm{H}), 5.11(\mathrm{~s}, 1 \mathrm{H}) 4.66(\mathrm{~s}, 1 \mathrm{H}), 3.93,(\mathrm{~s}, 1 \mathrm{H}), 3.52(\mathrm{~s}, 3 \mathrm{H}) 2.58(\mathrm{~d}$, $J=14.1 \mathrm{~Hz}, 1 \mathrm{H}), 2.5(\mathrm{~d}, J=7.5 \mathrm{~Hz}, 1 \mathrm{H}), 2.36(\mathrm{~m}, 1 \mathrm{H}), 2.29(\mathrm{~d}, J=7.1 \mathrm{~Hz}, 1 \mathrm{H}), 2.08$ (dd, $J$ $=9.8,3.6 \mathrm{~Hz}, 1 \mathrm{H}), 2.0(\mathrm{~m}, 2 \mathrm{H}) 1.73(\mathrm{~s}, 3 \mathrm{H}), 1.43(\mathrm{~m}, 1 \mathrm{H}), 1.38(\mathrm{~m}, 1 \mathrm{H}), 0.91(\mathrm{~d}, J=6.8 \mathrm{~Hz}$, $3 \mathrm{H}), 0.78(\mathrm{~d}, J=6.8 \mathrm{~Hz}, 3 \mathrm{H}) .{ }^{13} \mathrm{C}-\mathrm{NMR}\left(125 \mathrm{MHz}, \mathrm{CDCl}_{3}\right): \delta 171.2,170.3,135.8,133.9$, 122.3, 117.2, 103.2, 81.5, 74.9, 51.5, 50.6, 47.0, 44.2, 42.3, 35.5, 29.0, 23.1, 21.5, 21.4, 21.0 . IR (film): 3800-3000 (bs), 2955, 2867, 1658, 1618, 1442, 1363, 1305, 1260, 1248, 1203, 1147, $1081 \mathrm{~cm}^{-1} \cdot[\alpha]^{20}{ }_{D}-78.9\left(c 1.2, \mathrm{CHCl}_{3}\right)$. HRMS calcd. for $\mathrm{C}_{20} \mathrm{H}_{28} \mathrm{O}_{4}[\mathrm{M}+\mathrm{Na}]^{+} 355.1885$, found 355.1879 .
\end{abstract}

\section{Preparation of $(1 R, 2 R, 6 R, 7 R, 8 R, 9 R)$-9-allyl-6-isopropyl-3-methyl-12-oxa-tricyclo[6.3.1.02,7] dodec-3-en-10-one (27)}

A round-bottom flask was charged with compound 33 (1.75 g, $5.27 \mathrm{mmol}), \mathrm{LiCl}(1.17 \mathrm{~g}, 26.3$ mmol, 5 equiv), DMSO $(100 \mathrm{~mL})$ and $\mathrm{H}_{2} \mathrm{O}(378 \mu \mathrm{L})$. The solution was warmed to $130{ }^{\circ} \mathrm{C}$ and stirred at this temperature for $4 \mathrm{~h}$. After cooling the mixture to rt, a saturated $\mathrm{NaCl}$ solution (35 $\mathrm{mL}$ ) was added. This mixture was extracted with $\mathrm{Et}_{2} \mathrm{O}(5 \times 20 \mathrm{~mL})$. The combined organic phases were washed with a concentrated $\mathrm{NaCl}$ solution, dried $\left(\mathrm{MgSO}_{4}\right)$ and filtered. The solvent was removed under reduced pressure. The residue was purified via column chromatography applying EtOAc-hexane (3\%) as eluent to provide compound 27 (1.24 g, 86\%). ${ }^{1} \mathrm{H}-\mathrm{NMR}\left(500 \mathrm{MHz}, \mathrm{CDCl}_{3}\right): \delta 5.74(\mathrm{~m}, 1 \mathrm{H}), 5.54(\mathrm{~s}, 1 \mathrm{H}), 5.09(\mathrm{~d}, J=15.1 \mathrm{~Hz}, 1 \mathrm{H})$, $5.06(\mathrm{~d}, J=9.3 \mathrm{~Hz}, 1 \mathrm{H}), 4.46(\mathrm{~d}, J=5.1 \mathrm{~Hz}, 1 \mathrm{H}), 4.15(\mathrm{~s}, 1 \mathrm{H}), 2.76(\mathrm{dd}, J=15.05 .1 \mathrm{~Hz}, 1 \mathrm{H})$, $2.43(\mathrm{~m}, 2 \mathrm{H}), 2.24$ (series of multiplets, $3 \mathrm{H}), 2.15(\mathrm{t}, J=6.5 \mathrm{~Hz}, 1 \mathrm{H}), 1.95(\mathrm{~d}, J=8.3 \mathrm{~Hz}, 1 \mathrm{H})$, $1.55(\mathrm{~m}, 1 \mathrm{H}), 1.66(\mathrm{~s}, 3 \mathrm{H}), 1.60(\mathrm{~m}, 1 \mathrm{H}), 1.32(\mathrm{~m}, 1 \mathrm{H}), 0.90(\mathrm{~d}, J=6.8 \mathrm{~Hz}, 3 \mathrm{H}), 0.76(\mathrm{~d}, J=$ $6.8 \mathrm{~Hz}, 3 \mathrm{H}) .{ }^{13} \mathrm{C}-\mathrm{NMR}\left(125 \mathrm{MHz}, \mathrm{CDCl}_{3}\right): \delta 209.9,134.7,132.3,122.9,117.4,82.2,78.6$, 57.7, 47.6, 47.0, 45.1, 41.4, 35.2, 28.9, 22.4, 22.1, 21.5, 18.1. IR (film): 3070, 2956, 2868, $1712,1442,1385,1184,1036 \mathrm{~cm}^{-1} \cdot[\alpha]^{20}{ }_{\mathrm{D}}-156.0\left(c 1.0, \mathrm{CHCl}_{3}\right)$. HRMS calcd. for $\mathrm{C}_{18} \mathrm{H}_{26} \mathrm{O}_{2}{ }^{+}[\mathrm{M}]^{+}$275.2011, found: 275.1997.

\section{Preparation of (1R,2R,6R,7R,8R,9R)-9-(3-hydroxy-propyl)-6-isopropyl-3-methyl-12-oxa- tricyclo[6.3.1.02,7]dodec-3-en-10-one (34)}

Compound 27 (770 mg, $2.81 \mathrm{mmol}$ ) was dissolved in THF (36 mL), and a solution of dicyclohexylborane ( $1 \mathrm{M}$ in THF, $2.81 \mathrm{~mL}, 1$ equiv) was added at rt under argon. The resulting solution was stirred for $30 \mathrm{~min}$ at rt. Subsequently, $\mathrm{NaBO}_{3} \cdot \mathrm{H}_{2} \mathrm{O}(1.29 \mathrm{~g}, 8.43 \mathrm{mmol}, 3$ equiv) and $\mathrm{H}_{2} \mathrm{O}(10 \mathrm{~mL})$ was added and the reaction was stirred for an additional $4 \mathrm{~h}$. Then the reaction mixture was diluted with $\mathrm{Et}_{2} \mathrm{O}(10 \mathrm{~mL})$, and the phases were separated. The aqueous phase was extracted with EtOAc $(3 \times 5 \mathrm{~mL})$. The combined organic phases were washed with a concentrated $\mathrm{NaCl}$ solution, dried $\left(\mathrm{MgSO}_{4}\right)$ and filtered. The solvent was removed under 
reduced pressure. The residue was purified via column chromatography applying EtOAchexane (20\%) as eluent to provide compound $\mathbf{3 4}(618 \mathrm{mg}, 75 \%$ based on recovered starting material). ${ }^{1} \mathrm{H}-\mathrm{NMR}\left(500 \mathrm{MHz}, \mathrm{CDCl}_{3}\right): \delta 5.56(\mathrm{~s}, 1 \mathrm{H}), 4.48(\mathrm{~d}, J=4.95 \mathrm{~Hz}, 1 \mathrm{H}), 4.15(\mathrm{~s}, 1 \mathrm{H})$, $3.65(\mathrm{t}, J=5.5 \mathrm{~Hz}, 2 \mathrm{H}), 2.81(\mathrm{dd}, J=15.0,5.2 \mathrm{~Hz}, 1 \mathrm{H}), 2.42-2.20$ (series of multiplets, $3 \mathrm{H}$ ), $2.16(\mathrm{t}, J=6.5 \mathrm{~Hz}, 1 \mathrm{H}), 1.95(\mathrm{~d}, J=15.0 \mathrm{~Hz}, 1 \mathrm{H}), 1.92-1.70$ (series of multiplets, $4 \mathrm{H}), 1.68$ $(\mathrm{s}, 3 \mathrm{H}), 1.68-1.51$ (series of multiplets, $3 \mathrm{H}), 1.31(\mathrm{~m}, 1 \mathrm{H}), 0.93(\mathrm{~d}, J=6.8 \mathrm{~Hz}, 3 \mathrm{H}), 0.78(\mathrm{~d}$, $J=6.8 \mathrm{~Hz}, 3 \mathrm{H}) .{ }^{13} \mathrm{C}-\mathrm{NMR}\left(125 \mathrm{MHz}, \mathrm{CDCl}_{3}\right): \delta 210.8,132.3,123.0,83.5,78.7,62.2,57.8$, 47.4, 46.8, 45.2, 41.5, 29.9, 28.9, 27.4, 22.4, 22.1, 21.6, 18.1. IR (film): 3600-3100 (bs), 3070,

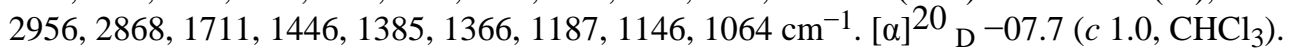
HRMS calcd. for $\mathrm{C}_{18} \mathrm{H}_{28} \mathrm{O}_{3}[\mathrm{M}+\mathrm{Na}]^{+} 315.1936$, found 315.1928.

\section{Preparation of (1R,2R,6R,7R,8R,9R)-9-(3-Chloro-propyl)-6-isopropyl-3-methyl-12-oxa- tricyclo[6.3.1.0 2,7 dodec-3-en-10-one (28)}

To a solution of compound 27 (450 mg, $1.54 \mathrm{mmol})$ in $\mathrm{CH}_{2} \mathrm{Cl}_{2}(30 \mathrm{~mL})$ was added $\mathrm{Ph}_{3} \mathrm{P}(504$ $\mathrm{mg}, 1.92 \mathrm{mmol}, 1.25$ equiv) under a nitrogen atmosphere at $\mathrm{rt}$. The resulting solution was cooled to $-10{ }^{\circ} \mathrm{C}$ and hexachloroacetone $(373 \mu \mathrm{L}, 2.46 \mathrm{mmol}, 1.6$ equiv) was added. The reaction was stirred at this temperature for $20 \mathrm{~min}$. Then $\mathrm{H}_{2} \mathrm{O}(10 \mathrm{~mL})$ was added and the phases were separated. The aqueous phase was extracted with $\mathrm{CH}_{2} \mathrm{Cl}_{2}(3 \times 5 \mathrm{~mL})$. The combined organic phases were washed with saturated $\mathrm{NaCl}$ solution, dried $\left(\mathrm{MgSO}_{4}\right)$ and filtered. The solvent was removed under reduced pressure. The resulting residue was purified via column chromatography applying EtOAc-hexane (10\%) as eluent to provide compound $28(470 \mathrm{mg}, 88 \%) .{ }^{1} \mathrm{H}-\mathrm{NMR}\left(500 \mathrm{MHz}, \mathrm{CDCl}_{3}\right):{ }_{-} 5.56(\mathrm{~d}, J=4.3 \mathrm{~Hz}, 1 \mathrm{H}), 4.48(\mathrm{~d}, J=5.0$ $\mathrm{Hz}, 1 \mathrm{H}), 4.12(\mathrm{~s}, 1 \mathrm{H}), 3.55(\mathrm{~m}, 2 \mathrm{H}), 2.81(\mathrm{dd}, J=15.0,5.2 \mathrm{~Hz}, 1 \mathrm{H}), 2.35(\mathrm{~d}, J=6.2 \mathrm{~Hz}, 1 \mathrm{H})$ $2.26(\mathrm{~d}, J=15.7 \mathrm{~Hz}, 1 \mathrm{H}), 2.24(\mathrm{t}, J=6.2 \mathrm{~Hz}, 1 \mathrm{H}), 2.17(\mathrm{t}, J=6.2 \mathrm{~Hz}, 1 \mathrm{H}) 1.97(\mathrm{~d}, J=15.7$ $\mathrm{Hz}, 1 \mathrm{H}$ ), 1.95-1.82 (series of multiplets, $3 \mathrm{H}$ ), 1.82-1.72 (series of multiplets, $2 \mathrm{H}$ ), $1.68(\mathrm{~s}, 3 \mathrm{H}$ ), $1.62(\mathrm{dd}, J=15.0,6.2 \mathrm{~Hz}, 1 \mathrm{H}), 1.31(\mathrm{~m}, 1 \mathrm{H}), 0.92(\mathrm{~d}, J=6.8 \mathrm{~Hz}, 3 \mathrm{H}), 0.80(\mathrm{~d}, J=6.8 \mathrm{~Hz}$, 3H). ${ }^{13} \mathrm{C}-\mathrm{NMR}\left(125 \mathrm{MHz}, \mathrm{CDCl}_{3}\right)$ : _ 210.1, 132.4, 123.0, 83.7, 78.8, 57.5, 47.4, 46.9, 45.2, 44.3, 41.6, 29.9, 29.0, 28.2, 22.4, 22.1, 21.6, 18.2. IR (film): 3070, 2954, 2933, 2894, 2864, $1705,1409,1379,1292,1191,1128,1104,1083 \mathrm{~cm}^{-1} \cdot[\alpha]^{20}{ }_{\mathrm{D}}^{-108.5}\left(c 0.55, \mathrm{CHCl}_{3}\right)$. HRMS calcd. for $\mathrm{C}_{18} \mathrm{H}_{27} \mathrm{ClO}_{2}[\mathrm{M}]^{+} 333.1597$, found 333.1589.

\section{Preparation of (1R,2R,3S,4S,6R,7R,8R,9R)-4-bromo-9-(3-chloro-propyl)-3-hydroxy-6- isopropyl-3-methyl-12-oxa-tricyclo[6.3.1.02,7]dodecan-10-one (39)}

Compound 28 (450 mg, $1.45 \mathrm{mmol}$ ) was dissolved in a mixture of THF $(17.4 \mathrm{~mL})$ and $\mathrm{H}_{2} \mathrm{O}$ (3.2 mL). To this solution was added NBS (334 mg, $1.89 \mathrm{mmol}, 1.3$ equiv) in three portions over $1 \mathrm{~h}$ at $0{ }^{\circ} \mathrm{C}$ under nitrogen. The reaction was allowed to warm to rt and stirred for $4 \mathrm{~h}$. Then the mixture was diluted with $\mathrm{H}_{2} \mathrm{O}(3 \mathrm{~mL})$ and $\mathrm{Et}_{2} \mathrm{O}(5 \mathrm{~mL})$ The phases were separated and the aqueous phase was extracted with EtOAc $(3 \times 3 \mathrm{~mL})$. The combined organic phases were washed with a saturated $\mathrm{NaCl}$ solution, dried $\left(\mathrm{MgSO}_{4}\right)$ and filtered. The solvent was removed under reduced pressure. The resulting residue was purified via column chromatography applying EtOAc-hexane (20\%) as eluent to provide compound 39 (450 mg, 71\%). ${ }^{1} \mathrm{H}-\mathrm{NMR}\left(500 \mathrm{MHz}, \mathrm{CDCl}_{3}\right): \delta 4.81(\mathrm{~d}, J=4.5 \mathrm{~Hz}, 1 \mathrm{H}), 4.11(\mathrm{~m}, 2 \mathrm{H}), 3.53$ (t, $J=6.1$ $\mathrm{Hz}, 2 \mathrm{H}), 2.81$ (dd, $J=15.5,5.0 \mathrm{~Hz}, 1 \mathrm{H}), 2.27$ (d, $J=15.5 \mathrm{~Hz}, 1 \mathrm{H}$ ), 2.12-2.07 (series of multiplets, $5 \mathrm{H}), 2.0(\mathrm{~m}, 1 \mathrm{H}), 1.9-1.6(\mathrm{~m}, 6 \mathrm{H}), 1.41(\mathrm{~s}, 3 \mathrm{H}), 0.91(\mathrm{~d}, J=6.8 \mathrm{~Hz}, 3 \mathrm{H}), 0.82(\mathrm{~d}$, $J=6.8 \mathrm{~Hz}, 3 \mathrm{H}) .{ }^{13} \mathrm{C}-\mathrm{NMR}\left(125 \mathrm{MHz}, \mathrm{CDCl}_{3}\right): \delta 209.5,84.2,77.5,72.3,62.8,57.0,53.2,47.8$, 44.9, 44.2, 39.7, 30.9, 30.3, 29.7, 28.3, 21.8, 21.2, 15.7. IR (film): 3600-3100, 2957, 2873, $1710,1453,1434,1410,1371,1349,1229,1171,1123,1037 \mathrm{~cm}^{-1} \cdot[\alpha]^{20}{ }_{\mathrm{D}}+20.3(c 1.5$, $\mathrm{CHCl}_{3}$ ). HRMS calcd. for $\mathrm{C}_{18} \mathrm{H}_{28} \mathrm{BrClO}_{3}[\mathrm{M}]^{+} 429.0808$, found 429.0824 . 


\section{Preparation of (1R,2R,3S,,6R,7R,8R,9R)-9-(3-chloro-propyl)-3-hydroxy-6-isopropyl-3- methyl-12-oxa-tricyclo[6.3.1.0 2,7 dodecan-10-one (40)}

To a solution of compound $39(450 \mathrm{mg}, 1.10 \mathrm{mmol})$ and AIBN $18 \mathrm{mg}, 0.11 \mathrm{mmol})$ in benzene $(11 \mathrm{~mL})$ was added $n$-Bu $3 \mathrm{SnH}(355 \mu \mathrm{L}, 1.32 \mathrm{mmol}, 1.2$ equiv) under an argon atmosphere. The reaction was warmed to $90{ }^{\circ} \mathrm{C}$ and stirred at this temperature for $1 \mathrm{~h}$. Subsequently, the reaction was cooled to rt and the solvent was removed under reduced pressure. The resulting residue was purified via column chromatography applying EtOAc-hexane (20\%) as eluent to provide compound 40 (360 mg, 94). ${ }^{1} \mathrm{H}-\mathrm{NMR}\left(500 \mathrm{MHz}, \mathrm{CDCl}_{3}\right): \delta 4.31$ (bs, 1H), 4.01 (s, $1 \mathrm{H}), 3.53(\mathrm{~m}, 2 \mathrm{H}), 1.77(\mathrm{dd}, J=15,4.3 \mathrm{~Hz}, 1 \mathrm{H}), 2.31(\mathrm{~d}, J=15 \mathrm{~Hz}, 1 \mathrm{H}), 2.15(\mathrm{t}, J=6.7 \mathrm{~Hz}$, $1 \mathrm{H}) 2.08(\mathrm{dd}, J=10.0,8.0 \mathrm{~Hz}, 1 \mathrm{H}) 1.97(\mathrm{~m}, 1 \mathrm{H}), 1.88-1.73$ (series of multiplets, $3 \mathrm{H}), 1.66(\mathrm{~m}$, $1 \mathrm{H}), 1.62(\mathrm{~s}, 1 \mathrm{H}), 1.56(\mathrm{~m}, 1 \mathrm{H}), 1.47-1.25$ (series of multiplets, $4 \mathrm{H}), 1.24(\mathrm{~s}, 3 \mathrm{H}), 0.94(\mathrm{~d}, J=$ $6.8 \mathrm{~Hz}, 3 \mathrm{H}), 0.91(\mathrm{t}, J=7.3 \mathrm{~Hz}, 1 \mathrm{H}), 0.82(\mathrm{~d}, J=6.8 \mathrm{~Hz}, 3 \mathrm{H}) .{ }^{13} \mathrm{C}-\mathrm{NMR}\left(125 \mathrm{MHz}, \mathrm{CDCl}_{3}\right)$ : $\delta$ 210.2, 84.7, 76.5, 70.3, 55.3, 55.2, 50.1, 46.5, 44.8, 42.3, 35.4, 31.0, 30.3, 29.7, 29.6, 22.0, 18.4, 17.1. IR (film): 3600-3100, 2956, 2870, 1709, 1463, 1410, 1396, 1287, 1196, 1075 $\mathrm{cm}^{-1}$. $[\alpha]^{20}{ }_{\mathrm{D}}^{-6.93}\left(\mathrm{c} 1.6, \mathrm{CHCl}_{3}\right)$. HRMS calcd. for $\mathrm{C}_{18} \mathrm{H}_{29} \mathrm{ClO}_{3}-\mathrm{H}_{2} \mathrm{O}\left[\mathrm{M}-\mathrm{H}_{2} \mathrm{O}\right]^{+} 310.1699$, found 310.1695 .

\section{Preparation of $(1 R, 2 R, 3 S,, 6 R, 7 R, 8 R, 9 R)$-acetic acid 9-(3-chloro-propyl)-6-isopropyl-3- methyl-10-oxo-12-oxa-tricyclo[6.3.1.02,7]dodec-3-yl ester (41)}

To a solution of compound 40 (113 mg, $0.34 \mathrm{mmol})$ in $\mathrm{CH}_{2} \mathrm{Cl}_{2}(3.4 \mathrm{~mL})$ was added $\mathrm{Et}_{3} \mathrm{~N}(143$ $\mu \mathrm{L}, 1.03 \mathrm{mmol}, 3$ equiv), $\mathrm{Ac}_{2} \mathrm{O}$ (100 $\mu \mathrm{L}, 1.05 \mathrm{mmol}, 3.05$ equiv) and DMAP (17 mg, 0.14 mmol, 0.4 equiv). The reaction was stirred at $\mathrm{rt}$ for $6 \mathrm{~h}$. Addition of $\mathrm{Et}_{3} \mathrm{~N}$ and $\mathrm{Ac}_{2} \mathrm{O}$ was repeated twice. Then the reaction was cooled to $-10{ }^{\circ} \mathrm{C}$ and stirred overnight at this temperature. Upon completion of the reaction, $\mathrm{H}_{2} \mathrm{O}(5 \mathrm{~mL})$ was added and the phases were separated. The aqueous phase was extracted with $\mathrm{CH}_{2} \mathrm{Cl}_{2}(3 \times 2 \mathrm{~mL})$. The combined organic phases were washed with saturated $\mathrm{NaCl}$ solution and dried $\left(\mathrm{MgSO}_{4}\right)$. The solvent was evaporated under reduced pressure. The resulting residue was purified via column chromatography applying EtOAchexane (10\%) as eluent to provide compound 41 (65 mg, 66\% BRSM). ${ }^{1} \mathrm{H}-\mathrm{NMR}$ (500 MHz, $\left.\mathrm{CDCl}_{3}\right): \delta 4.34$ (bs, 1H), $3.99(\mathrm{~s}, 1 \mathrm{H}), 3.54(\mathrm{~m}, 2 \mathrm{H}), 2.78(\mathrm{dd}, J=15.0,4.3 \mathrm{~Hz}, 1 \mathrm{H}), 2.39(\mathrm{~d}$, $J=13.0 \mathrm{~Hz}, 1 \mathrm{H}), 2.33(\mathrm{~d}, J=15.5 \mathrm{~Hz}, 1 \mathrm{H}), 2.26(\mathrm{~m}, 1 \mathrm{H}), 2.16(\mathrm{~m}, 1 \mathrm{H}) 2.06(\mathrm{dd}, J=10.0,8.0$ $\mathrm{Hz} 1 \mathrm{H}), 1.95(\mathrm{~s}, 3 \mathrm{H}), 1.90-1.72(\mathrm{~m}, 4 \mathrm{H}), 1.64(\mathrm{~m}, 1 \mathrm{H}), 1.5(\mathrm{~s}, 3 \mathrm{H}), 1.45(\mathrm{~m}, 1 \mathrm{H}), 1.40-1.20$ (series of multiplets, $2 \mathrm{H}), 1.13(\mathrm{~m}, 1 \mathrm{H}), 0.94(\mathrm{~d}, J=6.8 \mathrm{~Hz}, 3 \mathrm{H}), 0.82(\mathrm{~d}, J=6.8 \mathrm{~Hz}$, 3H). ${ }^{13} \mathrm{C}-\mathrm{NMR}\left(125 \mathrm{MHz}, \mathrm{CDCl}_{3}\right): \delta 209.6,169.9,84.8,82.2,75.3,54.6,52.9,49.6,46.0$, 44.3, 42.0, 30.8, 30.5, 29.8, 29.3, 23.9, 22.3, 21.3, 18.3, 16.6. IR (film): 2956, 2872, 1728, $1712,1462,1443,1366,1258,1190,1145,1119 \mathrm{~cm}^{-1}$. $[\alpha]^{20}{ }_{\mathrm{D}}^{-13.4}\left(c 0.7, \mathrm{CHCl}_{3}\right)$. HRMS calcd. for $\mathrm{C}_{20} \mathrm{H}_{31} \mathrm{ClO}_{4}[\mathrm{M}+\mathrm{Na}]^{+}$393.1808, found 393.1824.

\section{Preparation of $(1 R, 2 R, 3 S, 6 R, 7 R, 8 R, 9 R)$-acetic acid 9-(3-iodo-propyl)-6-isopropyl-3- methyl-10-oxo-12-oxa-tricyclo[6.3.1.02,7]dodec-3-yl ester (26)}

To a solution of compound $41(75 \mathrm{mg}, 0.20 \mathrm{mmol})$ in acetone $(2 \mathrm{~mL})$ was added sodium iodide (304 mg, $2.02 \mathrm{mmol}, 10$ equiv). The reaction was warmed to $57^{\circ} \mathrm{C}$ and stirred for $14 \mathrm{~h}$ under nitrogen at this temperature. Upon completion, the reaction was cooled to $\mathrm{rt}$ and the solvent was removed under reduced pressure. The resulting residue was purified via column chromatography applying EtOAc-hexane (10\%) as eluent to provide compound 26 (83 mg, $89 \%$ BRSM).

${ }^{1} \mathrm{H}-\mathrm{NMR}\left(500 \mathrm{MHz}, \mathrm{CDCl}_{3}\right.$ ): $\delta 4.35(\mathrm{bs}, 1 \mathrm{H}), 3.98(\mathrm{~s}, 1 \mathrm{H}), 3.18(\mathrm{t}, J=6.25 \mathrm{~Hz}, 2 \mathrm{H}), 2.79$ (dd, $J=15.0,3.5 \mathrm{~Hz}, 1 \mathrm{H}), 2.40(\mathrm{~d}, J=13 \mathrm{~Hz}, 1 \mathrm{H}), 2.34(\mathrm{~d}, J=15.5 \mathrm{~Hz}, 1 \mathrm{H}), 2.27(\mathrm{~d}, J=4.45 \mathrm{~Hz}$, $1 \mathrm{H}), 2.16(\mathrm{~m}, 1 \mathrm{H}), 2.06(\mathrm{dd}, J=10.25,8.0 \mathrm{~Hz}, 1 \mathrm{H}), 1.95(\mathrm{~s}, 3 \mathrm{H}), 1.90-1.78$ (series of multiplets, $3 \mathrm{H}), 1.65(\mathrm{~m}, 1 \mathrm{H}), 1.59(\mathrm{~m}, 1 \mathrm{H}), 1.51(\mathrm{~s}, 3 \mathrm{H}), 1.56(\mathrm{~m}, 1 \mathrm{H}), 1.39-1.25$ (series of multiplets, $2 \mathrm{H}), 1.14(\mathrm{dt}, J=15.6,3.5 \mathrm{~Hz}, 1 \mathrm{H}), 0.95(\mathrm{~d}, J=6.8 \mathrm{~Hz}, 3 \mathrm{H}), 0.82(\mathrm{~d}, J=6.8 \mathrm{~Hz}, 3 \mathrm{H}) .{ }^{13} \mathrm{C}-$ NMR (125 MHz, $\left.\mathrm{CDCl}_{3}\right): \delta$ 209.5, 169.9, 84.8, 82.2, 75.3, 54.4, 52.9, 49.6, 46.0, 44.3, 42.0, 
32.8, 30.8, 30.5, 23.9, 22.3, 21.3, 18.3, 16.6, 5.7. IR (film): 2955, 2872, 1728, 1711, 1462 , $1442,1366,1257,1191,1118 \mathrm{~cm}^{-1}[\alpha]^{20}{ }_{\mathrm{D}}-14.5\left(c 0.4, \mathrm{CHCl}_{3}\right)$. HRMS calcd. for $\mathrm{C}_{20} \mathrm{H}_{31} \mathrm{IO}_{4}[\mathrm{M}+\mathrm{Na}]^{+}$485.1164, found 485.1154 .

\section{Preparation of tetracyclic quaternary alcohol 25}

Preparation of the $\mathrm{SmI}_{2}$ solution: To a slurry of $\mathrm{Sm}(59 \mathrm{mg}, 0.39 \mathrm{mmol}, 3.6$ equiv) in THF (2.4 $\mathrm{mL}$ ) was added $\mathrm{CH}_{2} \mathrm{I}_{2}(26 \mu \mathrm{L}, 0.33 \mathrm{mmol}, 3$ equiv) in two portions, dropwise, under an argon atmosphere at rt. The solution was stirred for $4 \mathrm{~h}$. Then the solution was cooled to $0{ }^{\circ} \mathrm{C}$ and HMPA ( $420 \mu \mathrm{L}, 1.98 \mathrm{mmol}, 18$ equiv) was added. The solution was then stirred for $15 \mathrm{~min}$ at $0{ }^{\circ} \mathrm{C}$. Procedure for the $\mathrm{SmI}_{2}$-mediated cyclization: A solution of compound $\mathbf{2 6}$ (52 mg, 0.11 $\mathrm{mmol})$ in THF $(1.1 \mathrm{~mL})$ was added dropwise via cannula to the $\mathrm{SmI}_{2}$ solution. The reaction was allowed to warm to $\mathrm{rt}$ and stirred for $1 \mathrm{~h}$. Upon completion, the reaction was quenched with Rochelle's salt solution $(1 \mathrm{~mL})$ and the mixture was stirred at $\mathrm{rt}$ for $20 \mathrm{~min}$. This was followed by the addition of $\mathrm{H}_{2} \mathrm{O}(2 \mathrm{~mL})$ and $\mathrm{Et}_{2} \mathrm{O}(3 \mathrm{~mL})$. The phases were separated and the aqueous phase was extracted with EtOAc $(3 \times 1.5 \mathrm{~mL})$. The combined organic phases were washed with a saturated $\mathrm{NaCl}$ solution, dried $\left(\mathrm{MgSO}_{4}\right)$ and filtered. The solvent was evaporated under reduced pressure. The resulting residue was purified via column chromatography applying EtOAc-hexane (20\%) as eluent to provide compound 25 (32 mg, 88\%). ${ }^{1} \mathrm{H}$-NMR $\left(500 \mathrm{MHz}, \mathrm{CDCl}_{3}\right): \delta 4.09(\mathrm{bs}, 1 \mathrm{H}), 3.78(\mathrm{~s}, 1 \mathrm{H}), 3.27(\mathrm{~d}, J=8.4,1 \mathrm{H}), 2.25-2.13$ (series of multiplets, $2 \mathrm{H}$ ), 2.08-1.97 (series of multiplets, $2 \mathrm{H}$ ), 1.97 (s, 3H), 1.80-1.56 (series of multiplets, 9H), 1.54 (s, 3H), 1.52-1.38 (series of multiplets, $2 \mathrm{H}$ ), 1.30-1.12 (series of multiplets, $2 \mathrm{H}), 0.92(\mathrm{~d}, J=6.8 \mathrm{~Hz}, 3 \mathrm{H}), 0.82(\mathrm{~d}, J=6.8 \mathrm{~Hz}, 3 \mathrm{H}) .{ }^{13} \mathrm{C}-\mathrm{NMR}(125 \mathrm{MHz}$, $\mathrm{CDCl}_{3}$ ): $\delta 70.3,83.8,83.5,77.5,74.7,50.1,48.8,46.3,45.0,44.5,42.0,31.3,31.2,30.9,23.7$, 22.6, 22.5, 21.4, 18.5, 16.5. IR (film): 3600-3100 (bs), 2952, 2871, 1728, 1442, 1383, 1252, $1148 \mathrm{~cm}^{-1} \cdot[\alpha]^{20}{ }_{\mathrm{D}}+40.5\left(c 0.2, \mathrm{CHCl}_{3}\right)$. HRMS calcd. for $\mathrm{C}_{20} \mathrm{H}_{32} \mathrm{O}_{4}[\mathrm{M}]^{+} 359.2198$, found 359.2196 .

\section{Preparation of tetracyclic alkene 24}

To a solution of compound $25(55 \mathrm{mg}, 0.164 \mathrm{mmol})$ in $\mathrm{MeCN}(1.6 \mathrm{~mL})$ was added the Burgess reagent ${ }^{3}(86 \mathrm{mg}, 0.36 \mathrm{mmol}, 2.2$ equiv) under a nitrogen atmosphere. The solution was warmed to $70{ }^{\circ} \mathrm{C}$ and stirred for $40 \mathrm{~min}$. Upon completion, the reaction mixture was allowed to cool to rt and $\mathrm{H}_{2} \mathrm{O}(1 \mathrm{~mL})$ was added. The phases were separated and the aqueous phase was extracted with $\mathrm{Et}_{2} \mathrm{O}(3 \times 0.5 \mathrm{~mL})$. The combined organic phases were washed with a saturated $\mathrm{NaCl}$ solution, dried $\left(\mathrm{MgSO}_{4}\right)$ and filtered. The solvent was removed under reduced pressure. The resulting residue was purified via column chromatography applying $\mathrm{Et}_{2} \mathrm{O}$-hexane (10\%) solvent system as eluent to provide compound $24(45 \mathrm{mg}, 88 \%)$. ${ }^{1} \mathrm{H}-\mathrm{NMR}(500 \mathrm{MHz}$, $\left.\mathrm{CDCl}_{3}\right): \delta 4.14(\mathrm{bs}, 1 \mathrm{H}), 4.10(\mathrm{~s}, 1 \mathrm{H}), 2.59(\mathrm{~d}, J=16.5 \mathrm{~Hz}, 1 \mathrm{H}), 2.46(\mathrm{~m}, 1 \mathrm{H}), 2.40(\mathrm{~d}, J=14$ $\mathrm{Hz}, 1 \mathrm{H}), 2.35-2.25$ (series of multiplets, $3 \mathrm{H}), 2.21(\mathrm{~m}, 1 \mathrm{H}), 2.12(\mathrm{t}, J=8.5,1 \mathrm{H}), 1.97(\mathrm{~s}, 3 \mathrm{H})$, 1.88 (series of multiplets, $2 \mathrm{H}), 1.74(\mathrm{~d}, J=16.5 \mathrm{~Hz}, 1 \mathrm{H}), 1.66(\mathrm{~m}, 1 \mathrm{H}), 1.55(\mathrm{~s}, 3 \mathrm{H}), 1.48-1.32$ (series of multiplets, 2H), $1.27(\mathrm{t}, J=13 \mathrm{~Hz}, 1 \mathrm{H}), 1.12(\mathrm{t}, J=13 \mathrm{~Hz}, 1 \mathrm{H}), 0.95(\mathrm{~d}, J=6.5 \mathrm{~Hz}$, $3 \mathrm{H}), 0.84(\mathrm{~d}, J=6.5 \mathrm{~Hz}, 3 \mathrm{H}) .{ }^{13} \mathrm{C}-\mathrm{NMR}\left(125 \mathrm{MHz}, \mathrm{CDCl}_{3}\right): \delta 170.1,137.8,131.5,83.4,80.4$, 74.5, 53.2, 48.2, 41.8, 36.2, 34.9, 32.3, 31.2, 30.7, 24.6, 22.4, 22.2, 21.3, 19.0, 17.0. IR (film): 2956, 2872, 1725, 1442, 1366, 1247, 1185, 1143, $1040 \mathrm{~cm}^{-1}$. $[\alpha]^{20}{ }_{\mathrm{D}}-8.66\left(c \mathrm{co}, \mathrm{CHCl}_{3}\right)$. HRMS calcd. for $\mathrm{C}_{20} \mathrm{H}_{30} \mathrm{O}_{3}[\mathrm{M}]^{+}$318.2194, found 318. 2190.

\section{Preparation of $(1 R, 2 S, 3 R, 6 R, 7 R, 8 R)$-acetic acid 6-isopropyl-3-methyl-9,13-dioxo-15-oxa- tricyclo[6.6.1.02,7]pentadec-3-yl ester (23)}

A solution of compound $24(45 \mathrm{mg}, 0.14 \mathrm{mmol})$ in $\mathrm{CH}_{2} \mathrm{Cl}_{2}(1.4 \mathrm{~mL})$ was cooled to $-78{ }^{\circ} \mathrm{C}$ and $\mathrm{O}_{3}$ was bubbled through the solution until a faint blue color persisted (approximately 10 seconds). The solution was purged with argon at $-78{ }^{\circ} \mathrm{C}$ for tem min to remove excess $\mathrm{O}_{3}$ then $\mathrm{Me}_{2} \mathrm{~S}(100 \mu \mathrm{L})$ was added. The reaction was stirred overnight at $\mathrm{rt}$. The solvent was evaporated 
under reduced pressure and the resulting residue was purified via column chromatography applying EtOAc-hexane (30\%) solvent system as eluent to provide compound $\mathbf{2 3}$ (40 mg, $83 \%$ ). ${ }^{1} \mathrm{H}-\mathrm{NMR}\left(500 \mathrm{MHz}, \mathrm{C}_{6} \mathrm{D}_{6}\right): \delta 3.90(\mathrm{dd}, J=9.5,6.5 \mathrm{~Hz}, 1 \mathrm{H}), 3.83(\mathrm{~s}, 1 \mathrm{H}), 2.96(\mathrm{dd}, J$ $=12.0,5.5 \mathrm{~Hz}, 1 \mathrm{H}), 2.87(\mathrm{dd}, J=11.5,6.5 \mathrm{~Hz} 1 \mathrm{H}), 2.8(\mathrm{dd}, J=5.5,2.5 \mathrm{~Hz}, 1 \mathrm{H}), 2.69(\mathrm{~d}, J=$ $14.5 \mathrm{~Hz}, 1 \mathrm{H}), 2.59(\mathrm{t}, J=6.5,2.5 \mathrm{~Hz}, 1 \mathrm{H}), 2.47(\mathrm{t}, J=6.5 \mathrm{~Hz}, 1 \mathrm{H}), 2.21(\mathrm{q}, J=14 \mathrm{~Hz}, 1 \mathrm{H})$, $2.06(\mathrm{~d}, J=11.5 \mathrm{~Hz}, 1 \mathrm{H}), 2.00-1.85$ (series of multiplets, $2 \mathrm{H}), 1.59(\mathrm{~s}, 3 \mathrm{H}), 1.55-1.45(\mathrm{~m}, 1 \mathrm{H})$, 1.27-1.03 (series of multiplets, $3 \mathrm{H}), 0.98(\mathrm{~m}, 1 \mathrm{H}), 0.80(\mathrm{t}, J=6.5 \mathrm{~Hz}, 1 \mathrm{H}), 0.81(\mathrm{~d}, J=6.5 \mathrm{~Hz}$, $3 \mathrm{H}), 0.74,(\mathrm{~d}, J=6.5 \mathrm{~Hz}, 3 \mathrm{H}) .{ }^{13} \mathrm{C}-\mathrm{NMR}\left(125 \mathrm{MHz}, \mathrm{C}_{6} \mathrm{D}_{6}\right): \delta 210.7,210.1,169.4,88.4,81.2$, 78.4, 48.2, 48.0, 44.1, 40.3, 40.2, 39.5, 31.8, 30.0, 28.8, 24.5, 23.1, 21.7, 18.8, 15.5. IR (film): $2956,2872,1725,1442,1367,1241,1185,1143,1072,1017 \mathrm{~cm}^{-1} \cdot[\alpha]^{20}{ }_{\mathrm{D}}+50(c 0.15$, $\mathrm{CHCl}_{3}$ ). HRMS calcd. for $\mathrm{C}_{20} \mathrm{H}_{30} \mathrm{O}_{4}[\mathrm{M}+\mathrm{Na}]^{+}$373.1990, found: 373.1992 .

\section{Preparation of $(1 R, 2 S, 3 R, 6 R, 7 R, 8 R)$-acetic acid 6-isopropyl-3-methyl-9-methylene-13- oxo-15-oxa-tricyclo[6.6.1.0,7] pentadec-3-yl ester (29)}

Procedure for the preparation of the phosphorous ylide: To a solution of $\mathrm{Ph}_{3} \mathrm{PCH}_{3} \mathrm{Br}(214 \mathrm{mg}$, $0.625 \mathrm{mmol})$ in benzene $(5 \mathrm{~mL})$ was added $\mathrm{KO} t-\mathrm{Bu}(56 \mathrm{mg}, 0.5 \mathrm{mmol})$. The mixture was stirred at $40{ }^{\circ} \mathrm{C}$ for thirty min.

Procedure for the Wittig reaction: Compound $\mathbf{2 3}(21 \mathrm{mg}, 0.06 \mathrm{mmol})$ was dissolved in benzene $(600 \mu \mathrm{L})$, and the stock solution of the ylide $(700 \mu \mathrm{L}, 0.625 \mathrm{mmol}, 1.1$ equiv) was added under an argon atmosphere. The reaction was warmed to $80^{\circ} \mathrm{C}$ and was stirred for $30 \mathrm{~min}$. The addition of the stock solution was repeated. The reaction was monitored by thin layer chromatography. Upon consumption of the starting material, the reaction was quenched with a saturated $\mathrm{NaCl}$ solution $(1 \mathrm{~mL})$. The phases were separated and the aqueous phase was extracted with benzene $(2 \times 0.5 \mathrm{~mL})$ and EtOAc $(2 \times 0.5 \mathrm{~mL})$. The combined organic phases were dried $\left(\mathrm{MgSO}_{4}\right)$ filtered and the solvent was removed under reduced pressure. The resulting residue was purified via column chromatography applying $\mathrm{Et}_{2} \mathrm{O}$-hexane (10\%) solvent system as eluent to provide compound $29(16 \mathrm{mg}, 77 \%) .{ }^{1} \mathrm{H}-\mathrm{NMR}(500 \mathrm{MHz}$, $\left.\mathrm{CDCl}_{3}\right): \delta 4.96(\mathrm{~s}, 1 \mathrm{H}), 4.90(\mathrm{~s}, 1 \mathrm{H}), 4.25(\mathrm{~s}, 1 \mathrm{H}), 5.15(\mathrm{t}, J=6.5 \mathrm{~Hz}, 1 \mathrm{H}) 3.25(\mathrm{dd}, J=11.5$, $6.0 \mathrm{~Hz}, 1 \mathrm{H}$ ), 2.85-2.75 (series of multiplets, $2 \mathrm{H}$ ), 2.63 (d, $J=14 \mathrm{~Hz}, 1 \mathrm{H}), 2.39$ (dd, $J=11.5$, $6.5 \mathrm{~Hz}, 1 \mathrm{H}), 2.34(\mathrm{~d}, J=11.5 \mathrm{~Hz}, 1 \mathrm{H}), 2.30-2.15$ (series of multiplets, $2 \mathrm{H}$ ), 2.20-2.05 (series of multiplets, $2 \mathrm{H}) 2.00,(\mathrm{~s}, 3 \mathrm{H}), 1.75(\mathrm{~m}, 1 \mathrm{H}), 1.50(\mathrm{~s}, 3 \mathrm{H}), 1.48,(\mathrm{~m}, 1 \mathrm{H}) 1.39-1.08$ (series of multiplets, $4 \mathrm{H}), 0.94(\mathrm{~d}, J=6.5 \mathrm{~Hz}, 3 \mathrm{H}), 0.78(\mathrm{~d}, J=6.5 \mathrm{~Hz}, 3 \mathrm{H}) .{ }^{13} \mathrm{C}-\mathrm{NMR}(125 \mathrm{MHz}$, $\left.\mathrm{CDCl}_{3}\right): \delta 213.5,169.9,148.4,113.7,85.2,82.4,76.7,48.5,48.3,44.4,42.1,40.7,34.1,29.7$, 28.7, 26.0, 24.4, 22.4, 21.6, 18.5, 15.6. IR (film): 2956, 2929, 2872, 1729, 1705, 1367, 1245, $1144,1045,1020 \mathrm{~cm}^{-1}[\alpha]^{20}{ }_{\mathrm{D}}+7.0\left(c 0.1, \mathrm{CHCl}_{3}\right)$. HRMS calcd. for $\mathrm{C}_{21} \mathrm{H}_{32} \mathrm{O}_{4}[\mathrm{M}]^{+}$ 348.2300 , found 348.2298 .

\section{Preparation of (1R,2S,3R,6R,7R,8R,13R)-acetic acid 13-hydroxy-6-isopropyl-3,13-dimethyl-9- methylene-15-oxa-tricyclo[6.6.1.0 2,7]pentadec-3-yl ester (30)}

Procedure for the preparation of the $\mathrm{Yb}(\mathrm{OTf})_{3} \cdot \mathrm{MeLi}$ solution: $\mathrm{Yb}(\mathrm{OTf})_{3}(285 \mathrm{mg}, 0.46 \mathrm{mmol})$ was dissolved in THF $(4.6 \mathrm{~mL})$ under an argon atmosphere. The slurry was stirred for $15 \mathrm{~min}$ at $\mathrm{rt}$ then it was cooled to $-78^{\circ} \mathrm{C}$. This was followed by the addition of MeLi (1 M in hexane, $460 \mu \mathrm{L}, 0.46 \mathrm{mmol}$ ) and the resulting deep red solution was stirred at this temperature for 30 $\min$.

Procedure for methyl addition to carbonyl: Compound $29(11 \mathrm{mg}, 0.031 \mathrm{mmol})$ was dissolved in THF $(310 \mu \mathrm{L})$ and the resulting solution was cooled to $-78^{\circ} \mathrm{C}$ under an argon atmosphere. To this mixture an excess of the $\mathrm{Yb}(\mathrm{OTf})_{3} \cdot \mathrm{MeLi}$ stock solution was added until a faint red color persisted. The reaction was followed by thin layer chromatography. Upon consumption of the starting material, the reaction was queched at $-78{ }^{\circ} \mathrm{C}$ with saturated $\mathrm{NaHCO}_{3}(400 \mu \mathrm{L})$. The mixture was allowed to warm to rt and was diluted with diethyl ether $(500 \mu \mathrm{L})$. The phases 
were separated and the aqueous phase was extracted with EtOAc $(3 \times 200 \mu \mathrm{L})$. The combined organic phases were washed with a saturated $\mathrm{NaCl}$ solution, dried $\left(\mathrm{MgSO}_{4}\right)$ and filtered. The solvent was removed under reduced pressure. The resulting residue was purified via column chromatography applying $\mathrm{Et}_{2} \mathrm{O}$-hexane (10\%) solvent system as eluent to provide compound 30 (9 mg, 80\%). ${ }^{1} \mathrm{H}-\mathrm{NMR}\left(500 \mathrm{MHz}, \mathrm{CDCl}_{3}\right): \delta 4.93(\mathrm{~s}, 1 \mathrm{H}), 4.87(\mathrm{~s}, 1 \mathrm{H}), 4.14(\mathrm{~s}, 1 \mathrm{H}), 4.04$ $(\mathrm{m}, 1 \mathrm{H}), 2.63(\mathrm{t}, J=5.5 \mathrm{~Hz}, 1 \mathrm{H}), 2.46-2.35$ (series of multiplets, $3 \mathrm{H}), 2.29$ (dd, $J=8.0,5.5$ $\mathrm{Hz}, 1 \mathrm{H}), 2.00(\mathrm{~s}, 3 \mathrm{H}), 1.99(\mathrm{~m}, 1 \mathrm{H}), 1.85(\mathrm{dd}, J=14.5,11.0 \mathrm{~Hz}, 1 \mathrm{H}), 1.77-1.71$ (series of multiplets, $2 \mathrm{H}), 1.53(\mathrm{~m}, 1 \mathrm{H}), 1.46(\mathrm{~s}, 3 \mathrm{H}), 1.43(\mathrm{~m}, 1 \mathrm{H}), 1.30-1.23$ (series of multiplets, $4 \mathrm{H}$ ), $1.20(\mathrm{~m}, 1 \mathrm{H}), 1.13(\mathrm{~s}, 3 \mathrm{H}), 0.94(\mathrm{~d}, J=7 \mathrm{~Hz}, 3 \mathrm{H}), 0.88(\mathrm{t}, J=7 \mathrm{~Hz}, 1 \mathrm{H}), 0.82,(\mathrm{~d}, J=7 \mathrm{~Hz}$, $3 \mathrm{H}) .{ }^{13} \mathrm{C}-\mathrm{NMR}\left(125 \mathrm{MHz}, \mathrm{CDCl}_{3}\right): \delta 170.1,152.3,110.9,85.3,82.6,76.1,73.1,53.8,47.3$, 43.5, 41.4, 41.06, 37.6, 31.3, 30.8, 29.6, 26.8, 24.2, 22.5, 21.6, 18.3, 16.2. IR (film): 3650-3000, $2951,2914,2851,1727,1470,1375,1250,1080 \mathrm{~cm}^{-1} \cdot[\alpha]^{20}{ }_{\mathrm{D}}+52.0\left(c 0.1, \mathrm{CHCl}_{3}\right)$. HRMS calcd. for $\mathrm{C}_{21} \mathrm{H}_{36} \mathrm{O}_{4}[\mathrm{M}+\mathrm{Na}]^{+}$387.2511, found 387.2498.

\section{Preparation of 3,7-diastereomer of polyanthellin A (31)}

To a solution of compound $\mathbf{3 0}(2.8 \mathrm{mg}, 0.0077 \mathrm{mmol})$ in THF $(300 \mu \mathrm{L})$ was added a solution of $\mathrm{Hg}\left(\mathrm{OCOCF}_{3}\right)(6.5 \mathrm{mg}, 0.0153 \mathrm{mmol}, 2$ equiv) in THF $(300 \mu \mathrm{L})$ under an argon atmosphere. The reaction mixture was warmed to $55^{\circ} \mathrm{C}$ and stirred at this temperature for $3 \mathrm{~h}$. Subsequently, the reaction was cooled to $\mathrm{rt}$ and $\mathrm{NaBH}_{4}(3.5 \mathrm{mg}, 0.092 \mathrm{mmol}$ (12 equiv) was added. The reaction was stirred for $30 \mathrm{~min}$. Then it was quenched with saturated $\mathrm{NaHCO}_{3}$ solution (500 $\mu \mathrm{L})$ and the mixture was diluted with $\mathrm{Et}_{2} \mathrm{O}(600 \mu \mathrm{L})$. The phases were separated and the aqueous phase was extracted with diethyl ether $(3 \times 200 \mu \mathrm{L})$. The combined organic phases were washed with a saturated $\mathrm{NaCl}$ solution, dried $\left(\mathrm{MgSO}_{4}\right)$ and filtered. The solvent was removed under reduced pressure. The resulting residue was purified via column chromatography applying $\mathrm{Et}_{2} \mathrm{O}$-hexane (10\%) solvent system as eluent to provide the 3,7diastereomer of polyanthellin A $(1.6 \mathrm{mg}, 60 \%) .{ }^{1} \mathrm{H}-\mathrm{NMR}\left(500 \mathrm{MHz}, \mathrm{CDCl}_{3}\right): \delta 4.046(\mathrm{t}, J=$ $5.5 \mathrm{~Hz}, 1 \mathrm{H}), 3.74(\mathrm{~s}, 1 \mathrm{H}), 3.26(\mathrm{t}, J=5.5 \mathrm{~Hz}, 1 \mathrm{H}), 2.24(\mathrm{dd}, J=14.0,5.5 \mathrm{~Hz}, 1 \mathrm{H}), 2.17-2.13$ (series of multiplets, $2 \mathrm{H}), 2.00(\mathrm{~s}, 3 \mathrm{H}), 1.85(\mathrm{~m}, 1 \mathrm{H}), 1.69-1.54$ (series of multiplets, $6 \mathrm{H}), 1.49$ (s, 3H), 1.46-1.35 (series of multiplets, $3 \mathrm{H}$ ), $1.25(\mathrm{~s}, 6 \mathrm{H}), 1.32-1.2$ (series of multiplets, $2 \mathrm{H}$ ), $0.94(\mathrm{~d}, J=7 \mathrm{~Hz}, 3 \mathrm{H}), 0.82(\mathrm{~d}, J=7 \mathrm{~Hz}, 3 \mathrm{H}) .{ }^{13} \mathrm{C}-\mathrm{NMR}\left(125 \mathrm{MHz}, \mathrm{CDCl}_{3}\right): \delta 170.1,152.3$, $110.9,85.3,82.6,76.1,73.153 .8,47.3,43.5,41.4,41.0,37.6,31.3,30.8,29.6,26.8,24.2,22.5$, 21.6, 18.3, 16.2. IR (film): 2951, 2914, 2851, 1727, 1462, 1443, 1257, 1076, 1013, 796 $\mathrm{cm}^{-1} \cdot[\alpha]^{20}{ }_{\mathrm{D}}+47.5\left(\mathrm{c} 0.05, \mathrm{CHCl}_{3}\right)$. HRMS calcd. for $\mathrm{C}_{22} \mathrm{H}_{36} \mathrm{O}_{4}[\mathrm{M}+\mathrm{H}]^{+} 365.2676$, found 365.2685 .

\section{Supplementary Material}

Refer to Web version on PubMed Central for supplementary material.

\section{Acknowledgements}

We acknowledge the National Institutes of Health, Merck Research Laboratories and Amgen for the generous support of this work. We are grateful to Eli Lilly and Company for the graduate fellowship for Barbara Czakó. We also thank Dr. George Furst for his assistance in performing the NOESY experiments.

\section{References}

1. Bernardelli P, Paquette LA. Heterocycles 1998;49:531-556.

2. Kennard O, Watson DG, Riva di Sanseverino L, Tursch B, Bosmans R, Djerassi C. Tetrahedron Lett 1968:2879-2884.

3. Bernardelli P, Moradei OM, Friedrich D, Yang J, Gallou F, Dyck BP, Doskotch RW, Lange T, Paquette LA. J Am Chem Soc 2001;123:9021-9032. [PubMed: 11552810]

4. Friedrich D, Doskotch RW, Paquette LA. Org Lett 2000;2:1879-1882. [PubMed: 10891181]

5. Friedrich D, Paquette LA. J Nat Prod 2002;65:126-130. [PubMed: 11858742] 
6. Gallou F, MacMillan DWC, Overman LE, Paquette LA, Pennington LD, Yang J. Org Lett 2001;3:135137. [PubMed: 11429857]

7. Paquette LA, Moradei OM, Bernardelli P, Lange T. Org Lett 2000;2:1875-1878. [PubMed: 10891180]

8. MacMillan DWC, Overman LE. J Am Chem Soc 1995;117:10391-10392.

9. Overman LE, Pennington LD. Org Lett 2000;2:2683-2686. [PubMed: 10990427]

10. MacMillan DWC, Overman LE, Pennington LD. J Am Chem Soc 2001;123:9033-9044. [PubMed: 11552811]

11. Corminboeuf O, Overman LE, Pennington LD. J Am Chem Soc 2003;125:6650-6652. [PubMed: 12769572]

12. Crimmins MT, Brown BH. J Am Chem Soc 2004;126:10264-10266. [PubMed: 15315437]

13. Molander GA, St Jean DJ Jr, Haas J. J Am Chem Soc 2004;126:1642-1643. [PubMed: 14871089]

14. Girard P, Namy JL, Kagan HB. J Am Chem Soc 1980;102:2693-2698.

15. Molander GA, Etter JB. Tetrahedron Lett 1984;25:3281-3284.

16. Molander GA, McKie JA. J Org Chem 1993;58:7216-7227.

17. Molander GA, Andrews SW. Tetrahedron Lett 1989;30:2351-2354.

18. Thap Do M, Strausz OP. J Am Chem Soc 1970;92:1766-1768.

19. Morton DR, Lee-Ruff E, Southam RM, Turro NJ. J Am Chem Soc 1970;92:4349-4357.

20. Morton DR, Turro NJ. J Am Chem Soc 1973;95:3947-3957.

21. Turro NJ, Morton DR. J Am Chem Soc 1971;93:2569-2571.

22. Krapcho AP, Glynn GA, Grenon BJ. Tetrahedron Lett 1967:215-217. [PubMed: 6037875]

23. Jefford CW, Sledeski AW, Lelandais P, Boukouvalas J. Tetrahedron Lett 1992;33:1855-1858.

24. Kabalka GW, Yu S, Li NS. Tetrahedron Lett 1997;38:5455-5458.

25. Kabalka GW, Yu S, Li NS. Can J Chem 1998;76:800-805.

26. Haas, J. PhD Thesis. University of Pennsylvania; Philadelphia: 2002. Application of the (4+3) Annulation Reactions to Natural Product Synthesis.

27. Krow GR. Org React 1993;43:251-798.

28. Cofre P, Sawyer DT. Inorg Chem 1986;25:2089-2092.

29. Mosome T, Atarashi S, Muraoka O. Tetrahedron Lett 1974;42:3697-3700.

30. Hunt KW, Grieco PA. Org Lett 2000;2:1717-1719. [PubMed: 10880209]

31. Hanessian S, Lavallee P. Can J Chem 1975;53:2975-2977.

32. Guss CO, Rosenthal R. J Am Chem Soc 1955;77:2549.

33. Kupchik EJ, Connolly RE. J Org Chem 1961;26:4747-4748.

34. Noltes JG, van der Kerk GJM. Chem Ind 1959:294.

35. Hoefle G, Steglich W, Vorbrueggen H. Angew Chem 1978;90:602-615.

36. Verheyden JPH, Moffatt JG. J Am Chem Soc 1964;86:2093-2095.

37. Machrouhi F, Hamann B, Namy JL, Kagan HB. Synlett 1996:633-634.

38. Spartan' 04, W., Molecular Mechanics Model, MMFF94 Forcefield, Conformation Distribution Calculation.

39. Magid RM, Fruchey OS, Johnson WL. Tetrahedron Lett 1977:2999-3002.

40. Finkelstein H. Ber 1910;43:1528-1532.

41. Burgess EM, Penton HR Jr, Taylor EA. J Am Chem Soc 1970;92:5224-5226.

42. Bailey PS. Chem Rev 1958;58:925-1010.

43. Wittig G, Schollkopf U. Chem Ber 1954;97:1318-1330.

44. According to MMFF calculations, the energy difference between the minimum energy conformation and the conformation where the ketone is rotated out is $5.35 \mathrm{kcal} / \mathrm{mol}$.

45. Molander GA, Burkhardt ER, Weinig P. J Org Chem 1990;55:4990-4991.

46. Broka CA, Lin YT. J Org Chem 1988;53:5876-5885. 


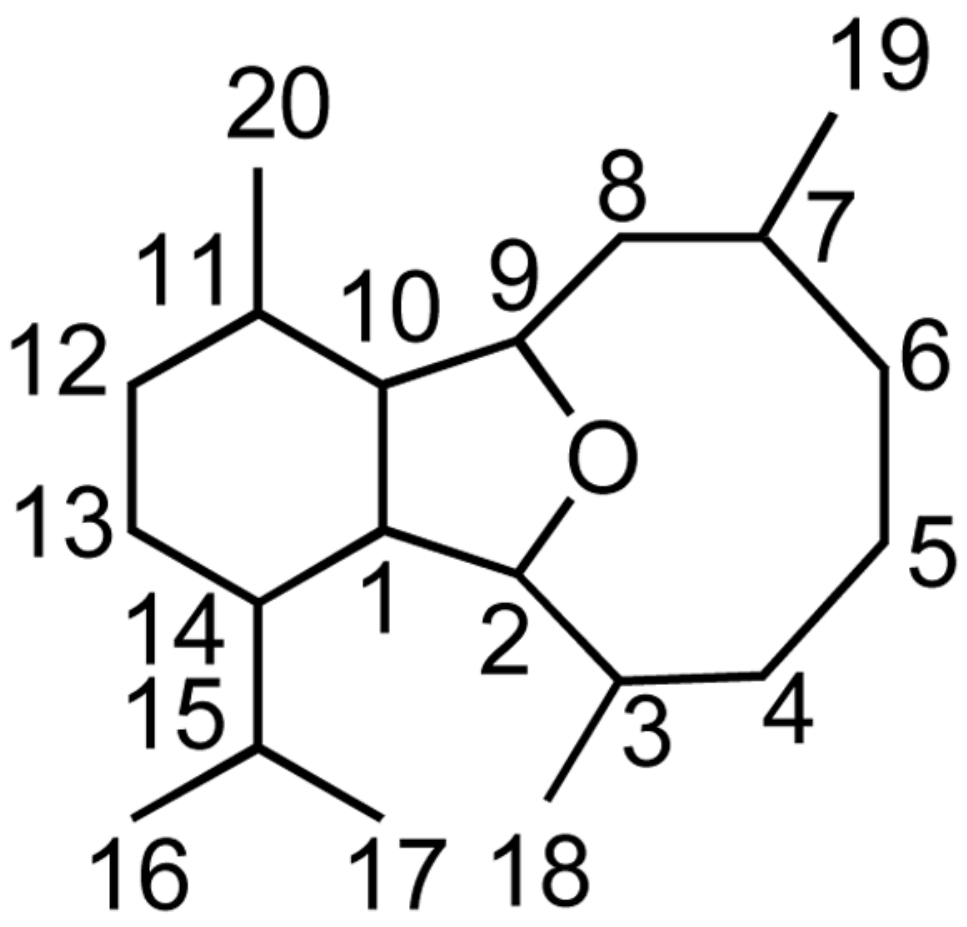

Figure 1.

Cladiellin skeleton. 

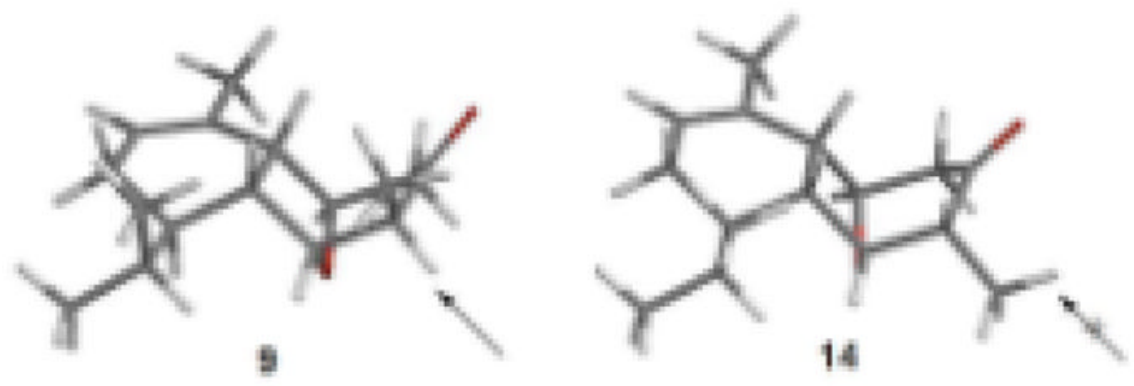

Figure 2.

3D representation of compound 9 and $\mathbf{1 4 .}$ 


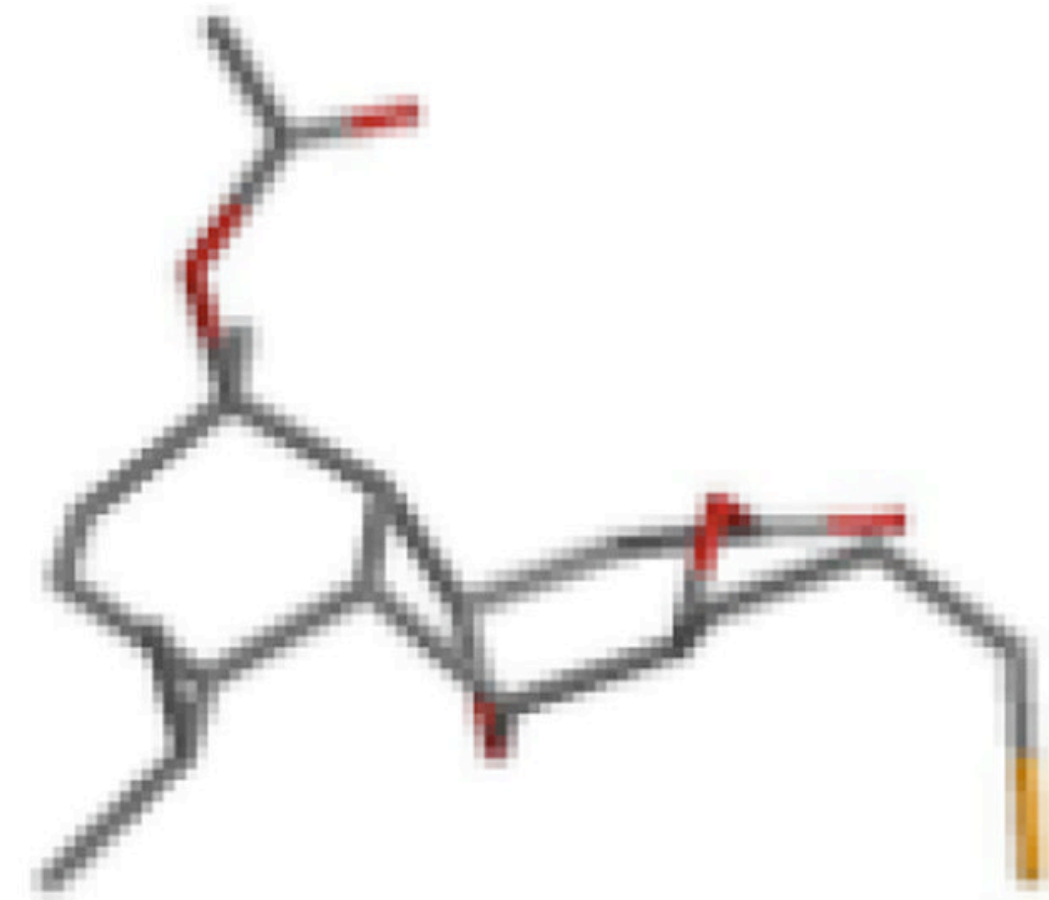

Figure 3.

Minimum energy conformation of iodolactone $\mathbf{1 7}$. 

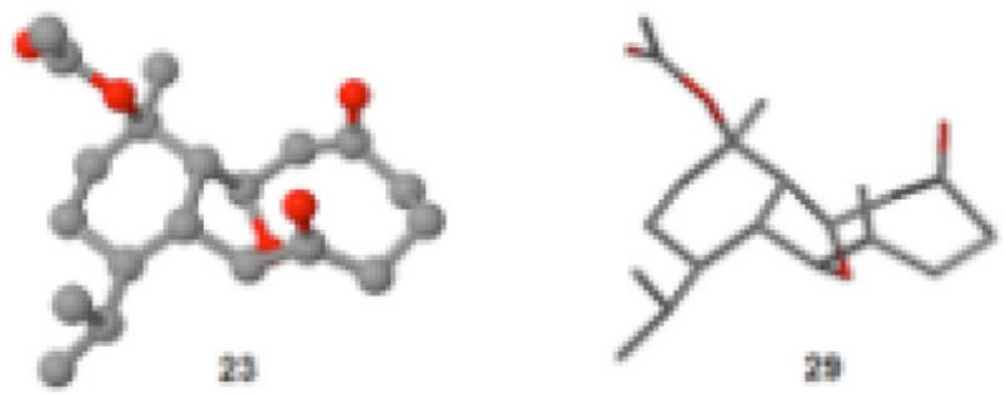

Figure 4.

$\mathrm{X}$-Ray structure of diketone $\mathbf{2 3}$ and minimum energy conformation of keto-alkene $\mathbf{2 9}$. 


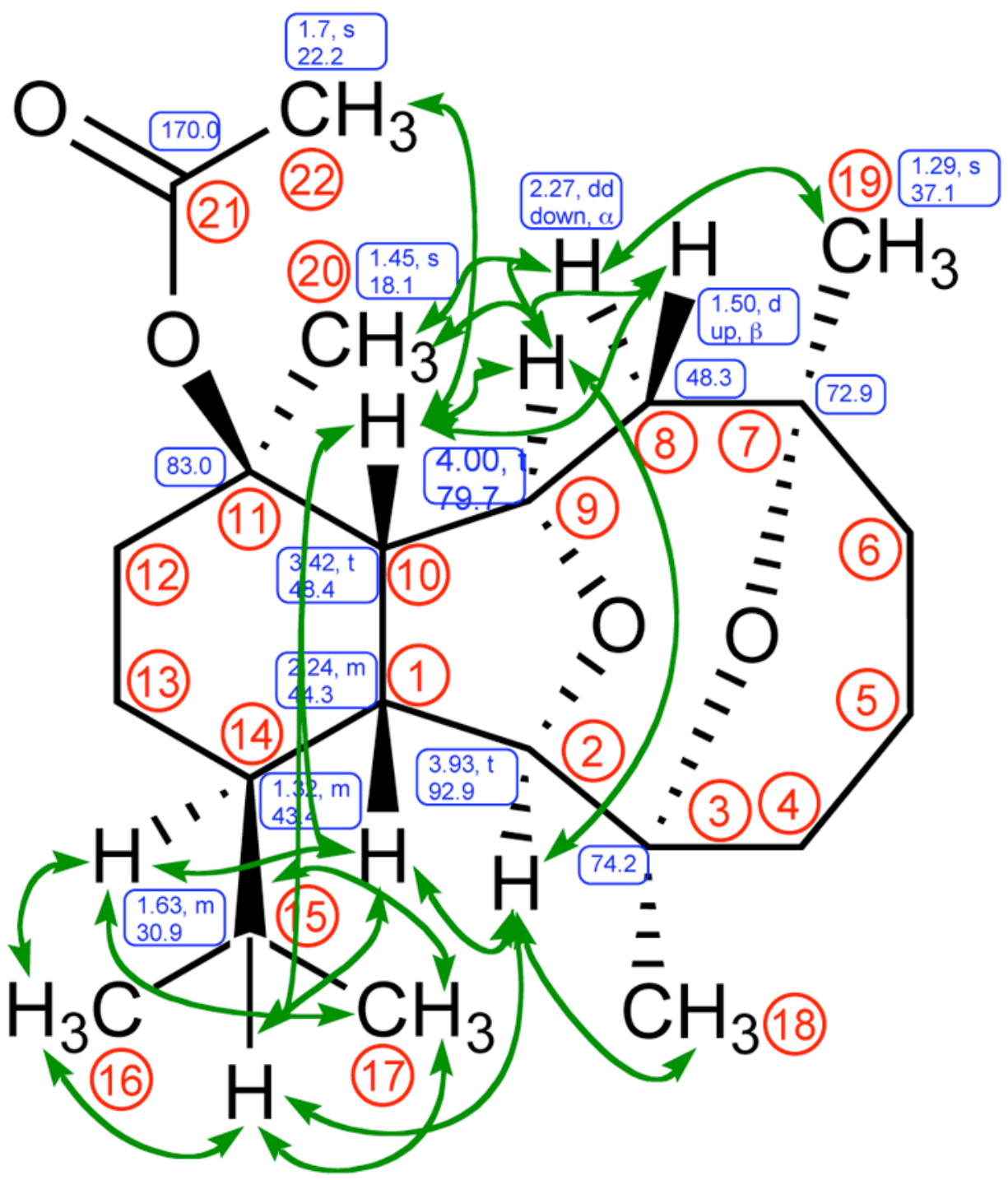

Figure 5.

NOESY correlation of the 3,7-diastereomer of polyanthellin A. 


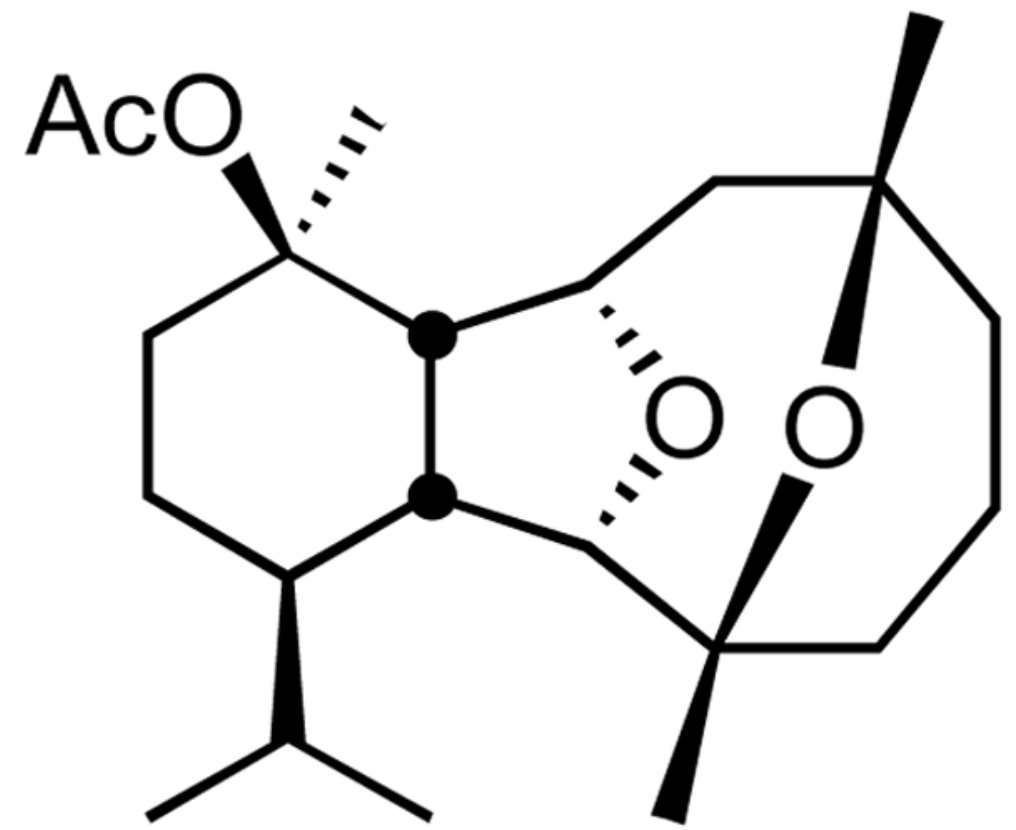

Figure 6.

Polyanthellin A. 

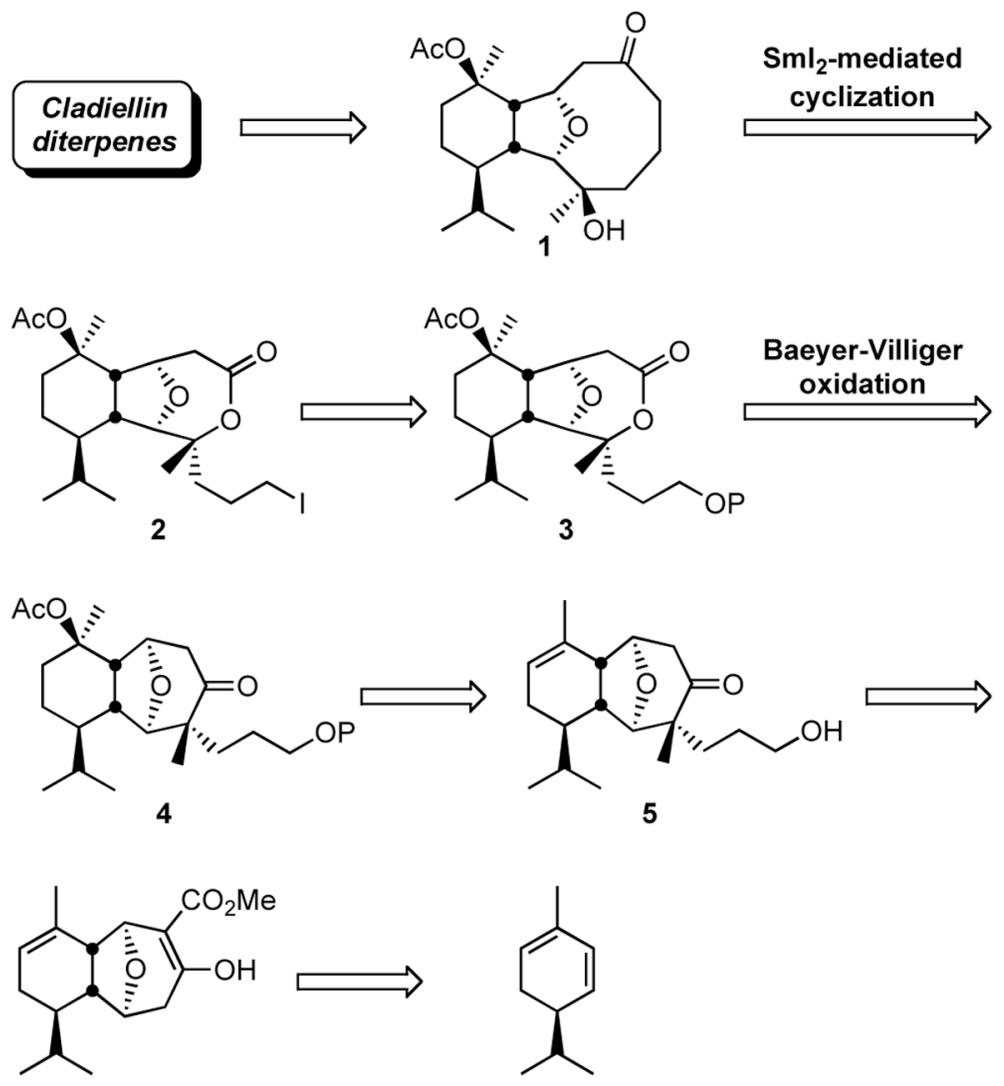

[4+3] annulation product

$\alpha$-Phellandrene 6

Scheme 1.

First synthetic approach to the cladiellin skeleton. 

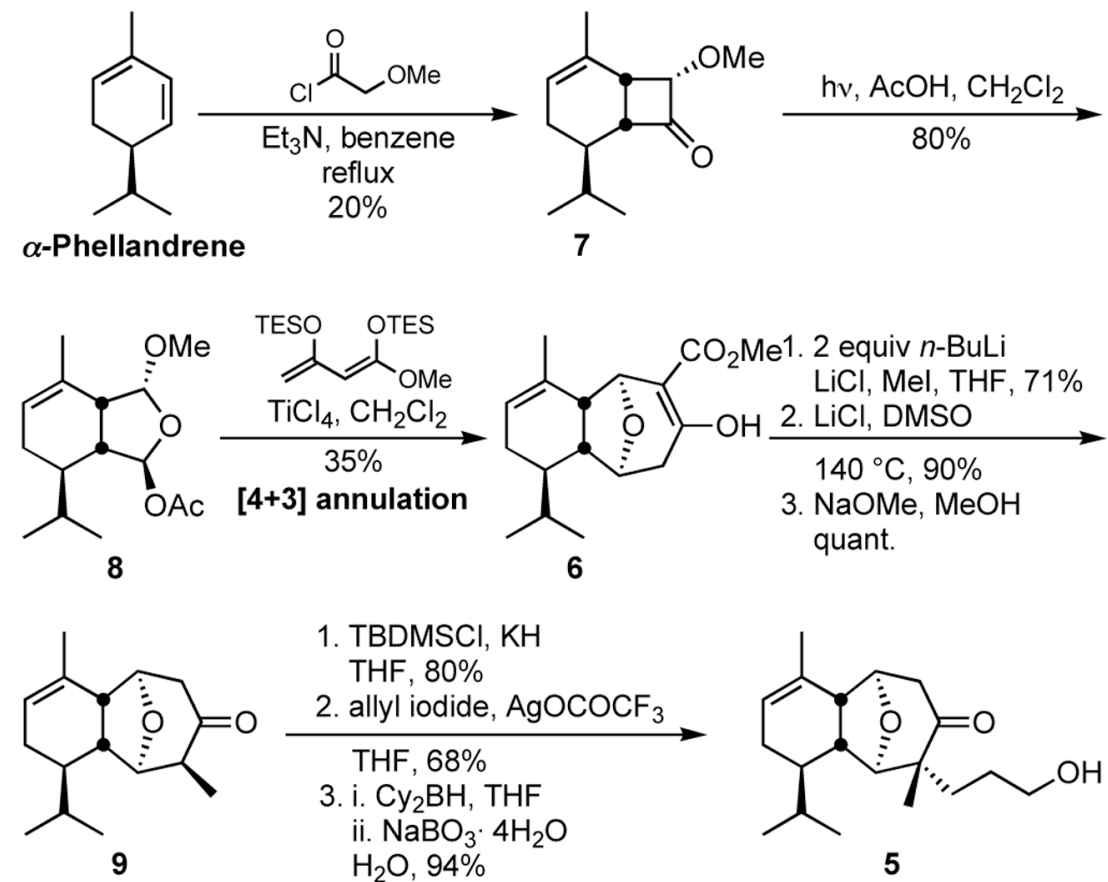

$$
\begin{aligned}
& \text { 1. TBDMSCl, } \mathrm{KH} \\
& \text { THF, } 80 \% \\
& \text { 2. allyl iodide, } \mathrm{AgOCOCF}_{3} \\
& \stackrel{\mathrm{THF}, 68 \%}{\text { THO }}, \\
& \text { 3. i. } \mathrm{Cy}{ }_{2} \mathrm{BH}, \mathrm{THF} \\
& \text { ii. } \mathrm{NaBO} \cdot 4 \mathrm{H}_{2} \mathrm{O} \\
& \mathrm{H}_{2} \mathrm{O}, 94 \%
\end{aligned}
$$

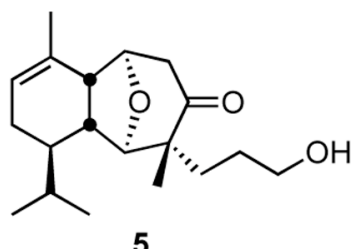

Scheme 2.

Initial steps of the synthesis. 

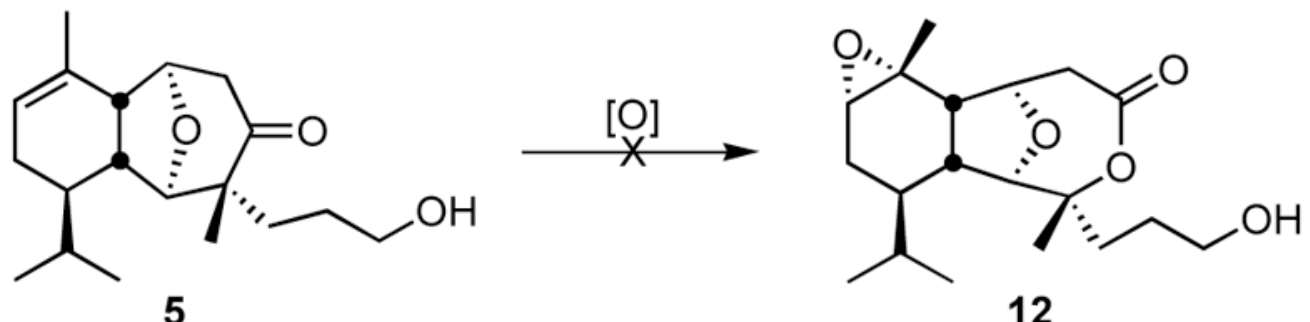

Scheme 3.

Baeyer-Villiger oxidation studies. 

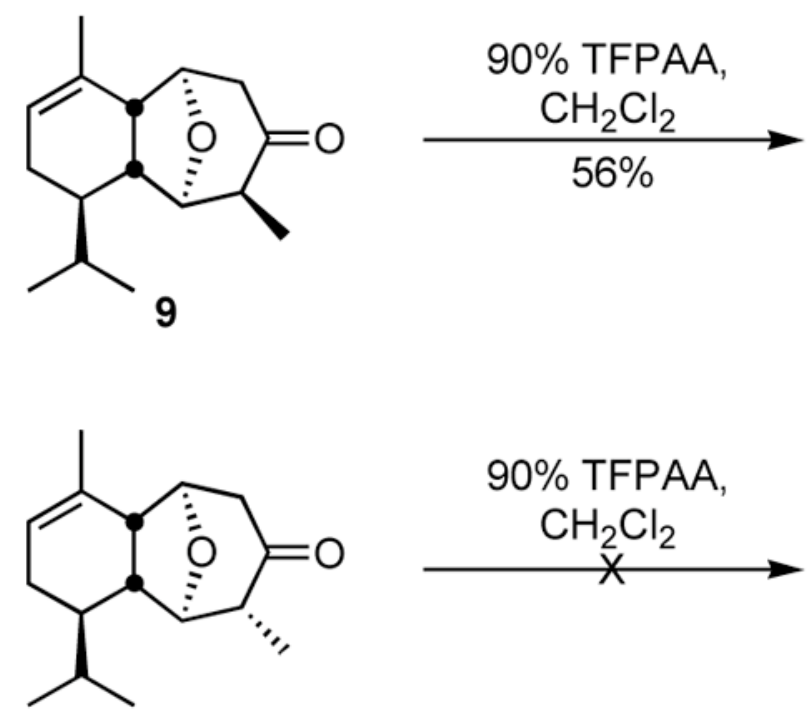

14

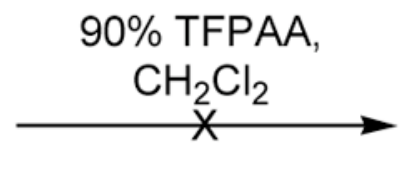

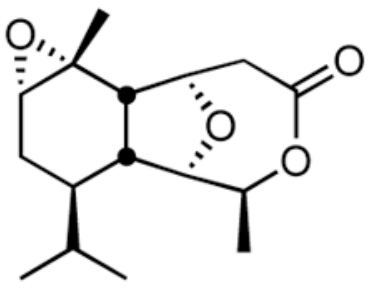

13

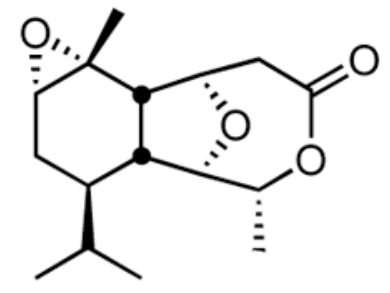

15

Scheme 4.

Baeyer-Villiger oxidation studies. 


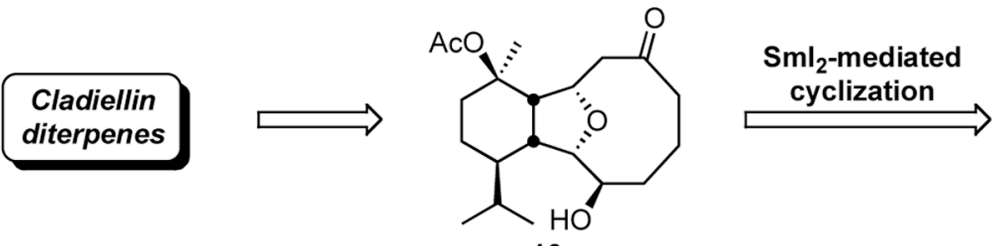

16
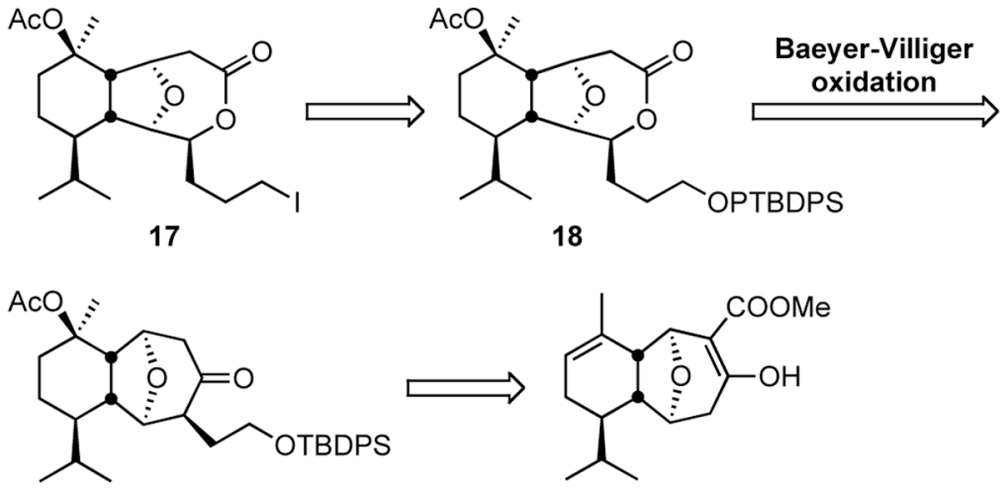

19

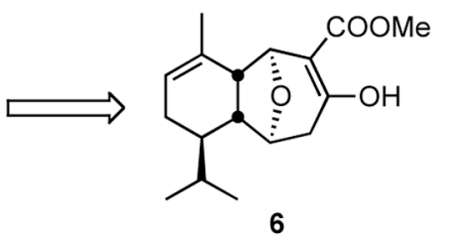

Scheme 5.

Second synthetic approach to the cladiellin skeleton. 


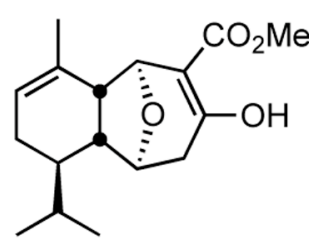

6
1. 2 equiv $n$-BuLi, $\mathrm{LiCl}$ allyl iodide, THF, 90\%

2. $\mathrm{LiCl}, \mathrm{DMSO}$, heat, $86 \%$

3. $\mathrm{NaOMe}, \mathrm{MeOH}$, heat $90 \%$ (3:1 mixture of diastereomers)

1. i. $\mathrm{Cy}_{2} \mathrm{BH}, \mathrm{THF}$ ii. $\mathrm{NaBO}_{3}, \mathrm{H}_{2} \mathrm{O}$ $65 \%$, brsm

2. TBDPSCl, imid. $\mathrm{CH}_{2} \mathrm{Cl}_{2}$, quant.

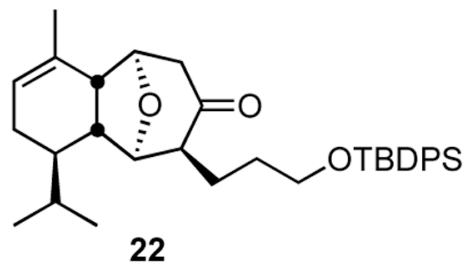

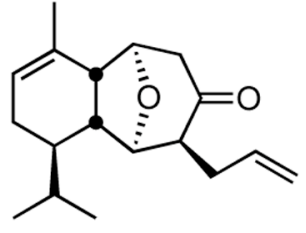

21

1. NBS, $\mathrm{H}_{2} \mathrm{O}$ THF, 98\% 2. $\mathrm{Bu}_{3} \mathrm{SnH}, \mathrm{AIBN}$ benzene, quant.

3. $\mathrm{Ac}_{2} \mathrm{O}, \mathrm{DMAP}$ $\mathrm{Et}_{3} \mathrm{~N}, \mathrm{CH}_{2} \mathrm{Cl}_{2}$ $88 \%$<smiles>CC(=O)O[C@@]1(C)CC[C@@H](C(C)C)[C@@H]2[C@H]1[C@H]1CC(=O)[C@H](CCCCl)[C@@H]2O1</smiles>

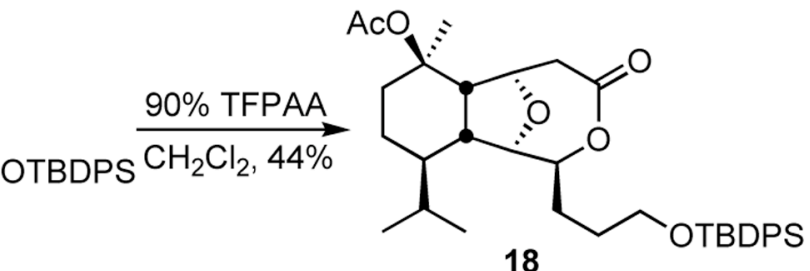

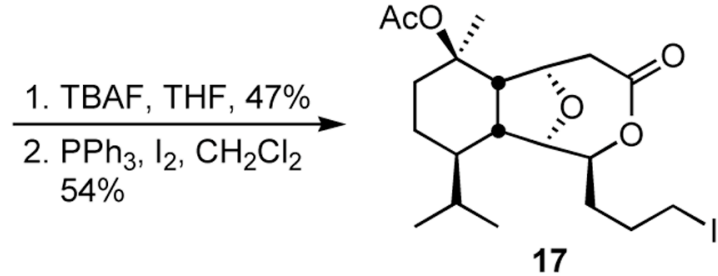

Scheme 6.

Synthesis of the $\mathrm{SmI}_{2}$-mediated cyclization precursor 17. 


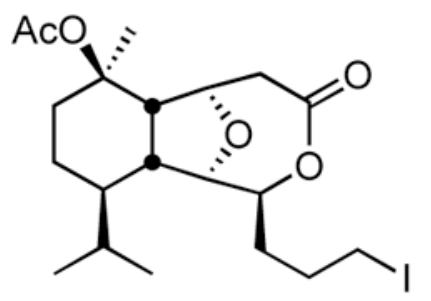

17

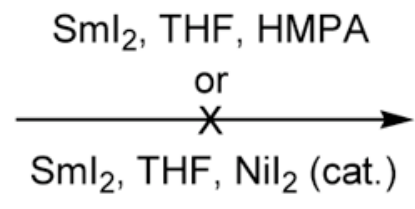

$\mathrm{Sml}_{2}, \mathrm{THF}, \mathrm{Nil}_{2}$ (cat.)

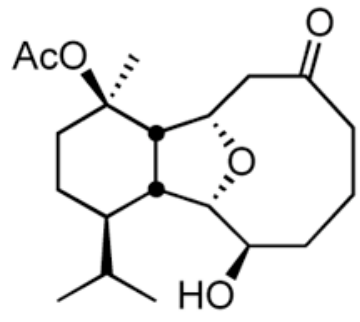

16

Scheme 7.

$\mathrm{SmI}_{2}$-mediated reaction. 


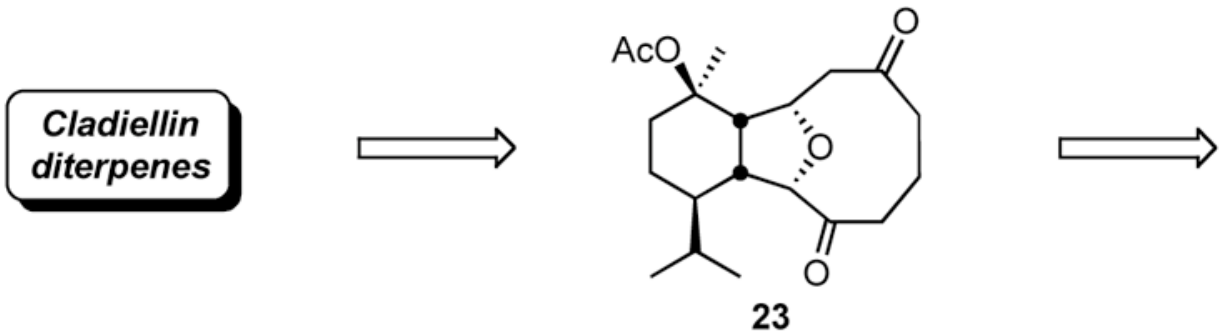

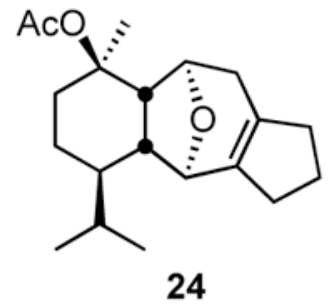

24

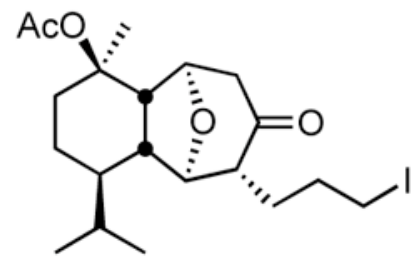

26

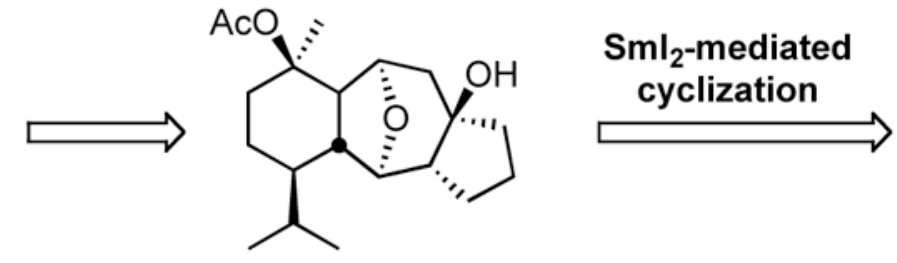

25
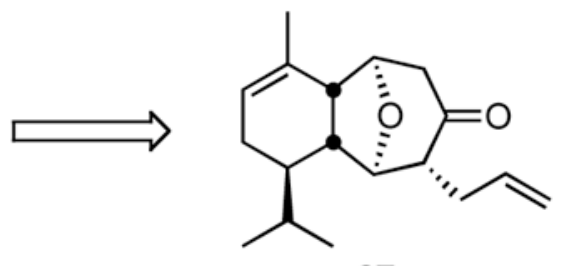

27

Scheme 8.

Third synthetic approach to the cladiellin skeleton. 


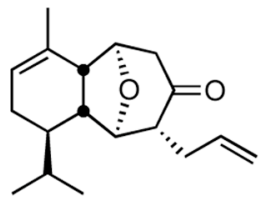

27

1. NBS, $\mathrm{H}_{2} \mathrm{O}$, THF, $71 \%$

2. $\mathrm{Bu}_{3} \mathrm{SnH}, \mathrm{AIBN}$ benzene, $94 \%$

3. $\mathrm{Ac}_{2} \mathrm{O}, \mathrm{DMAP}, \mathrm{Et}_{3} \mathrm{~N}$ $\mathrm{CH}_{2} \mathrm{Cl}_{2}, 66 \%$ brsm 4. Nal, acetone, $89 \%$<smiles>CC(=O)O[C@]1(C)CCC(C(C)C)[C@H]2[C@@H]3O[C@H](C[C@]4(O)CCC[C@H]34)[C@@H]21</smiles>

25

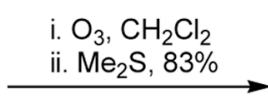

1. i. $\mathrm{Cy}_{2} \mathrm{BH}, \mathrm{THF}$

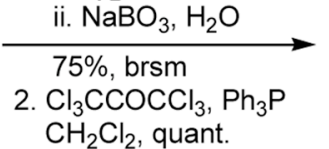
$\mathrm{CH}_{2} \mathrm{Cl}_{2}$, quant.

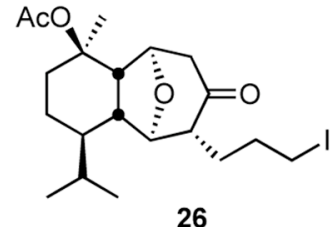

26
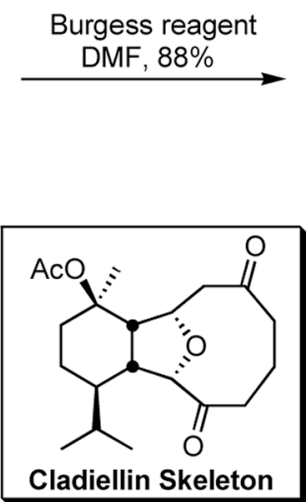

23

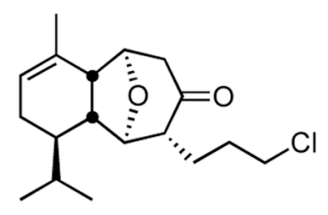

28
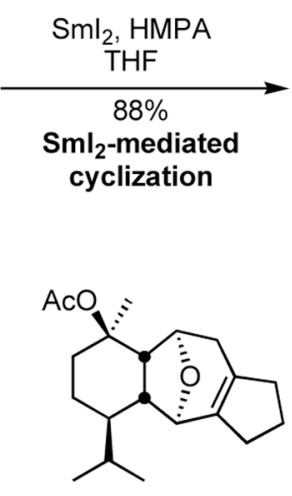

24

Scheme 9.

Synthesis of the cladiellin skeleton. 


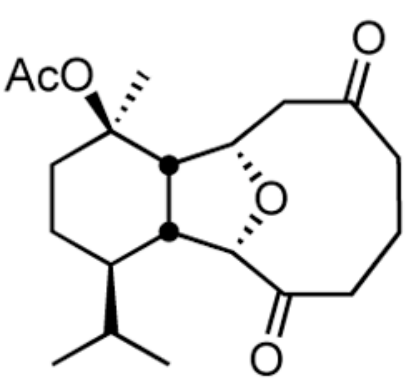

23

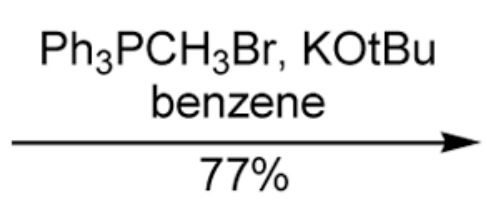

Scheme 10.

Chemoselective Wittig reaction of dicarbonyl 23.

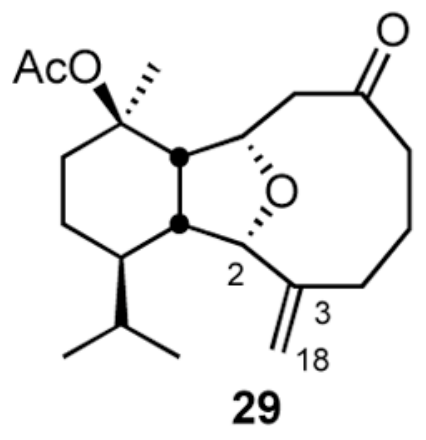




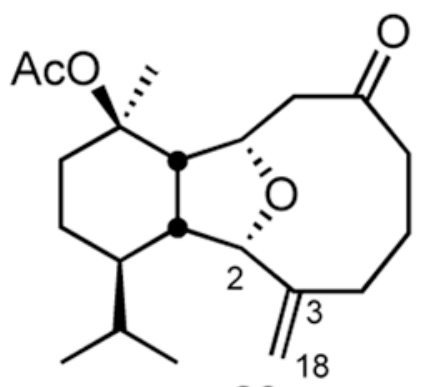

29

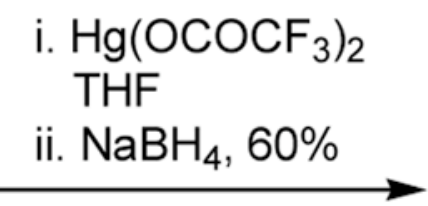

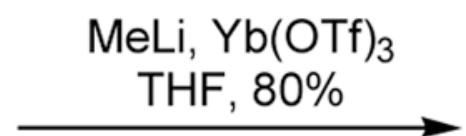
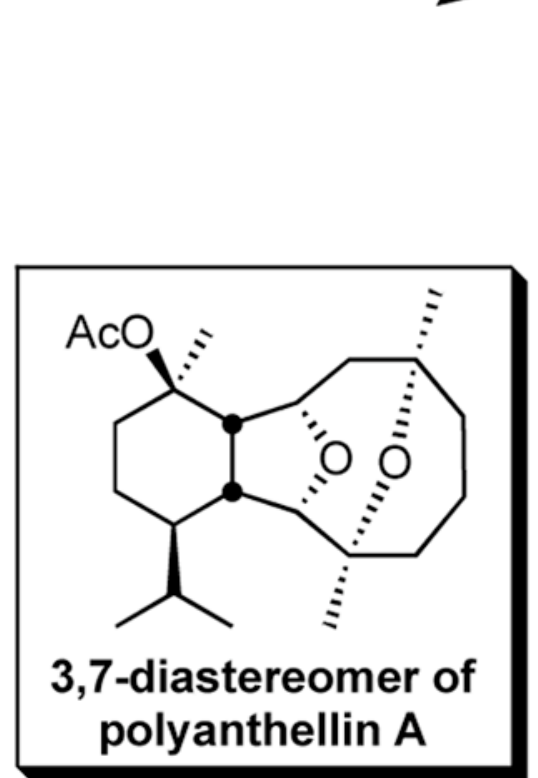

31

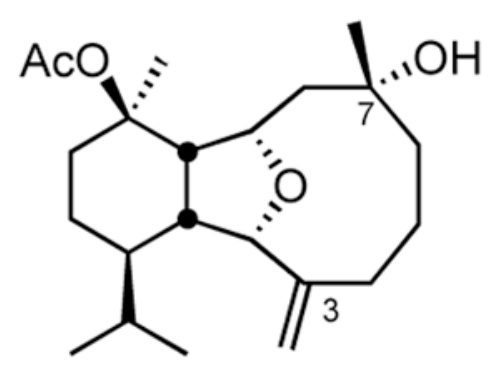

30

Scheme 11.

Synthesis of the 3,7-diastereomer of polyanthellin A. 